\title{
ESTUDIO DE LA ADAPTACIÓN A RDA Y BIBFRAME EN EL ÁMBITO DE LAS BIBLIOTECAS ESPAÑOLAS: ESTUDIOS DE CASO
}

\author{
Roberto Cormenzana López* \\ Universitat Oberta de Catalunya \\ Alexandre López-Borrull** \\ Universitat Oberta de Catalunya"
}

\begin{abstract}
Resumen: Actualmente, los usuarios prefieren acudir a motores de búsqueda para consultar la información que desean conocer, sin que las bibliotecas puedan jugar un papel protagónico, fundamentalmente porque los datos albergados en sus catálogos no se integran de forma natural y eficiente en la web. Para solventarlo, la comunidad bibliotecaria afronta una evolución en la forma de describir sus registros bibliográficos y de autoridad, planteando nuevos modelo conceptual (LRM), directrices de catalogación (RDA) y marco bibliográfico (BIBFRAME). Se ha realizado un estudio cualitativo del estado del arte, analizando artículos y conferencias sobre la materia y entablando entrevistas con representantes cualificados de una selección de instituciones bibliotecarias. Éstas aceptan su parte de responsabilidad y comienzan a abordar la implantación de RDA sobre MARC y a publicar sus catálogos en portales de datos abiertos enlazados. No obstante, la futura incorporación de BIBFRAME en detrimento de MARC será un paso más traumático y determinante.
\end{abstract}

Palabras clave: RDA; BIBFRAME; catalogación; descripción bibliográfica; bibliotecas; MARC; RDF; datos enlazados; web semántica; LRM; SIGB.

Title: STUDY OF THE ADAPTATION TO RDA AND BIBFRAME IN THE FIELD OF SPANISH LIBRARIES: CASE STUDIES.

Abstract: Currently, users prefer to go to search engines to consult the information they want to know, and libraries are not playing a key role in this, mainly because the data in their catalogs are not integrated natural and efficiently on the web. In order to solve it, the library community faces an evolution in the way of describing its bibliographic and authority records, and proposes a new conceptual model (LRM), new cataloging directives (RDA) and a new bibliographic framework (BIBFRAME). A qualitative study of the state of the art has been carried out, analyzing articles and conferences on the subject and interviewing qualified representatives of a selection of library institutions. They accept their role by undertaking the implementation of RDA on MARC and publishing their catalogs in Linked Open Data portals. However, the future incorporation of BIBFRAME to the detriment of MARC will be a more traumatic and decisive step.

Keywords: RDA; BIBFRAME; cataloging; bibliographic description; libraries; MARC; RDF; linked data; semantic web; LRM; ILS.

Copyright: (C) 2018 Servicio de Publicaciones de la Universidad de Murcia (Spain). Este es un artículo de acceso abierto distribuido bajo los términos de la licencia Creative Commons Reconocimiento 4.0 Internacional (CC BY 4.0).

\section{MARCO GENERAL}

\subsection{Escenario tradicional}

La práctica catalogadora desempeñada por la comunidad bibliotecaria ha disfrutado de cierta estabilidad a lo largo de más de cuatro décadas.

\footnotetext{
*rcormenzana@uoc.edu

**alopezbo@uoc.edu

Recibido: 3-03-2018; $2^{a}$ versión: 21-08-2018; aceptado: 25-09-2018.
}

CORMENZANA LÓPEZ, R. y LÓPEZ BORRULL, A. Estudio de la adaptación a RDA y BIBFRAME en el ámbito de las bibliotecas españolas: Estudios de caso. Anales de Documentación, 2018, vol. 21, $\mathrm{n}^{\circ} 2$. Disponible en: http://dx.doi.org/10.6018/analesdoc.21.2.323441. 


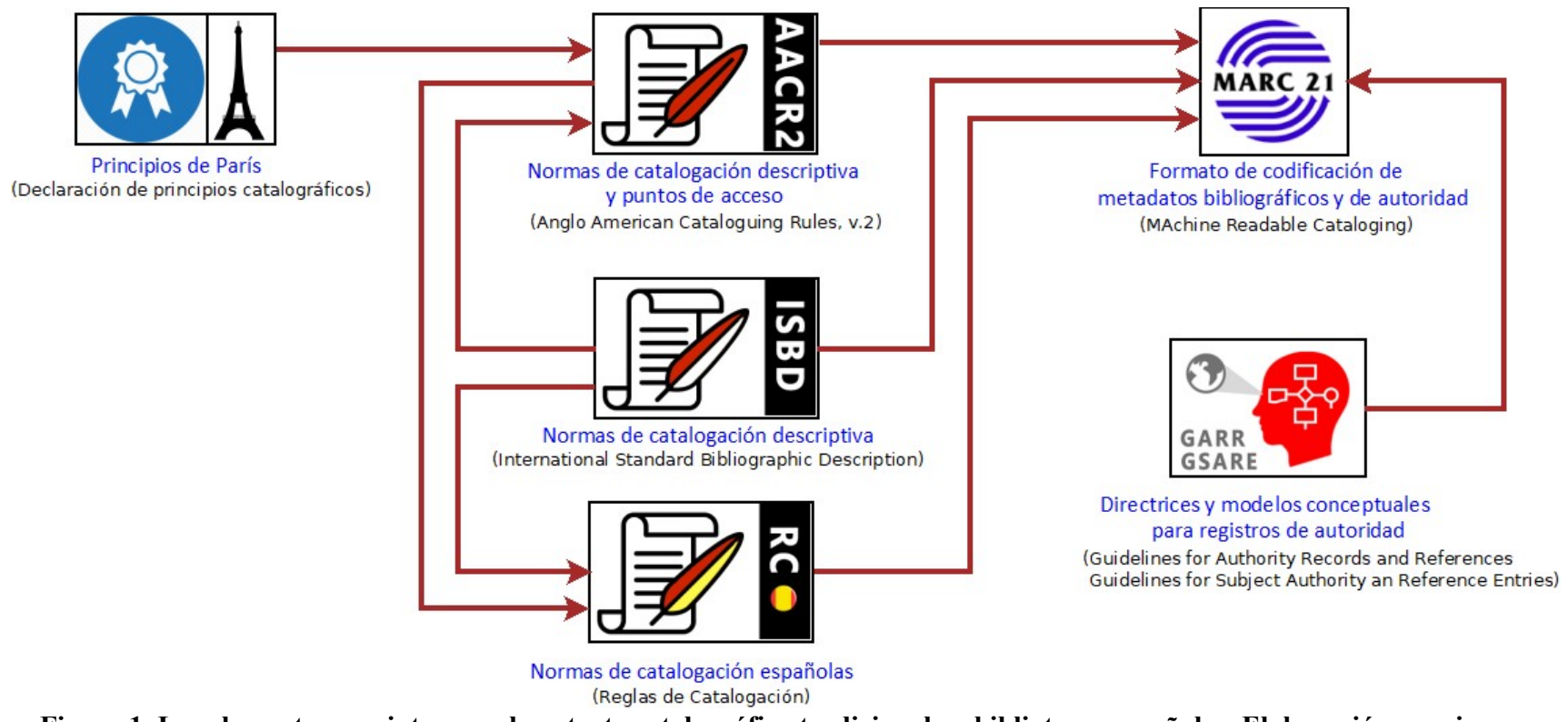

Figura 1. Los elementos que integran el contexto catalográfico tradicional en bibliotecas españolas. Elaboración propia.

La declaración primigenia de principios de catalogación, conocida como Principios de París, establecía qué funciones debe tener el catálogo, su estructura, los tipos de asientos que puede incluir, su elección y su cometido (IFLA, 2009). Las distintas reglas de catalogación se inspiran en estos para concretar las pautas para la creación de los registros bibliográficos y los puntos de acceso.

El mapa de aplicación de las reglas de catalogación en España tradicionalmente se ha dividido en dos grandes áreas, que han asumido diferentes aproximaciones estratégicas:

- Las bibliotecas que usan las RC (Reglas de Catalogación) españolas, que para la catalogación descriptiva se fundamenta en ISBD (International Standard Bibliographic Description). Son asumidas por gran parte de las bibliotecas españolas, y tuteladas y supervisadas por la Biblioteca Nacional de España (BNE).

- Las bibliotecas que apuestan por aplicar directamente las AACR2 (Anglo American Cataloging Rules, 2nd Edition), que incorpora tanto las normas de catalogación descriptiva como los puntos de acceso (Huthwaite, 2002). La Biblioteca de Cataluña (BC) es el principal referente de esta práctica bibliotecaria, que es asumida por la práctica totalidad de las bibliotecas catalanas.

Para determinar cómo debe ser la forma de los encabezamientos o puntos de acceso, las reglas de catalogación delegan en las directrices para la creación de registros de autoridad, tanto de referencia como explicativos. GARR (Guidelines for Authority Records and References) y GSARE (Guidelines for Subject Authority and Reference Entries) marcan las pautas para su estructura y presentación, pero no la elección y la construcción de estos, establecida por las propias normas de catalogación (BNE, 2016).

MARC (MAchine Readable Cataloguing) es un conjunto de formatos de codificación y comunicación de registros catalográficos legibles por el ordenador, utilizado en la mayoría de sistemas bibliotecarios automatizados. Se limita a especificar la estructura de los datos que se comunican entre sistemas de catalogación automatizados. De la identificación y descripción se encargan las normas de catalogación elegidas, y del almacenamiento y la visualización se encargará la aplicación de software de bibliotecas elegida por la organización (Furrie, 2009).

\subsection{La necesidad de un cambio}

En la actual sociedad de información, una gran proporción de usuarios buscan información primero en Google u otro buscador web, y eventualmente adquieren la documentación localizada en la biblioteca (a través del catálogo o mediante préstamo interbibliotecario). Los usuarios se consagran al "principio del mínimo esfuerzo", y en este sentido, la ecuación "información = web" se impone a la anterior y clásica "información = biblioteca" (Coyle, 2007).

Mientras que en los motores de búsqueda de la web se puede consultar rápida y fácilmente reseñas, portadas, citas, comentarios y valoraciones de otros usuarios y obras relacionadas, obteniéndose de forma integrada diversidad de información heterogénea relativa al criterio de búsqueda documental, los catálogos han encontrado grandes dificultades para formar parte de este contexto, y el contenido que albergan fundamentalmente ha permanecido circunscrito al ámbito informacional bibliotecario. De ahí su paulatina pérdida de protagonismo. 
Uno de los principales culpables de esta situación es MARC, ilustre estándar concebido por y para la comunidad bibliotecaria, inteligible y procesable solo dentro de este ámbito. Su uso ha dado pie a lo largo de los años a problemas de duplicidad, inconsistencia y complejidad en los datos (Alemu et al., 2012). La forma en que plasma su contenido, por medio de tablas organizadas en campos, etiquetas, indicadores y códigos de subcampo, lo hace inflexible y no apropiado para expresar relaciones asociativas, de pertenencia o jerárquicas, vital para el despliegue de información en el seno de internet.

Las arañas o indexadores web son incapaces de descifrar esos datos de forma nativa (Park y Morrison, 2017), y en todo caso su recolección estaría a expensas de la manera en que estos son interpretados y eventualmente publicados en internet por la heterogénea diversidad de sistemas de gestión o portales bibliotecarios capacitados para procesarlos. Estas carencias se hacen patentes en la práctica al consumar el formato MARC para registros bibliográficos su matrimonio con las reglas de catalogación de ISBD y AACR2, que por otra parte fueron concebidas muchos años antes del advenimiento de la web, y por tanto ajenas a los aspectos esenciales de los datos enlazados.

PRESENTE
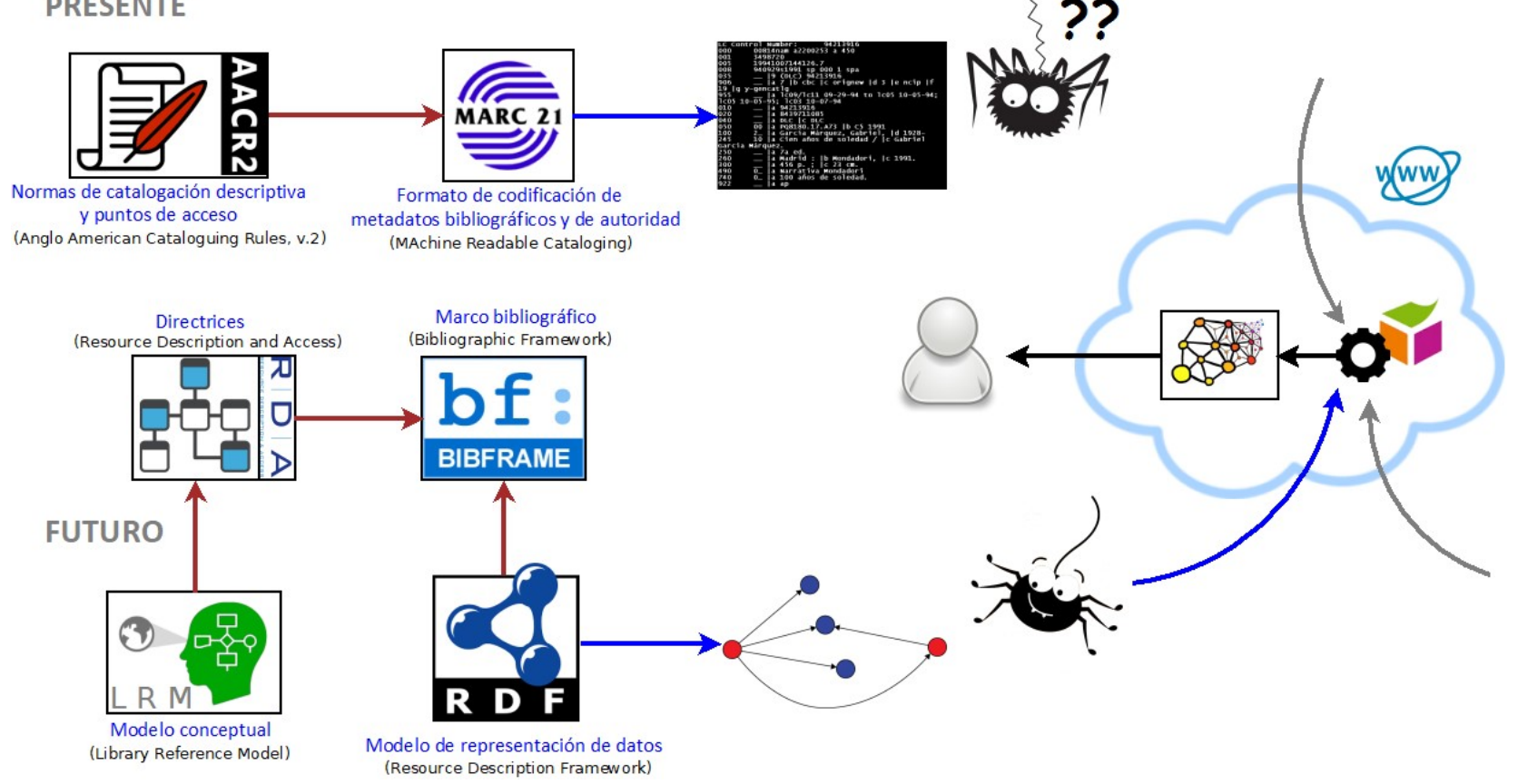

Figura 2. Relación con la web del antiguo y nuevo conjunto de especificaciones para catalogación. Elaboración propia.

La integración de MARC en los catálogos es tan profunda y su presencia tan abundante que su destierro en favor de nuevos estándares que sepan hablar como la web (Miller, 2015) no deja de tener tintes dramáticos tanto en términos económicos como técnicos. Miles de millones de registros, resultado de muchos años de prácticas de catalogación haciendo uso de ese formato, yacen en los catálogos de un ingente número de bibliotecas y permanecen aislados de internet, y que deben ser migrados a un contenedor que permita la publicación de su contenido como datos enlazados (Library of Congress, 2008).

\subsection{Escenario futuro}

El destierro de MARC y las antiguas reglas de catalogación para dar la bienvenida a un nuevo ecosistema de formatos y estándares para la generación, tratamiento y publicación de datos bibliográficos que mueva a las bibliotecas hacia la web es un proceso que ha de afrontarse de forma gradual, emprendiendo cambios parciales e iterativos, lo que hará que MARC todavía conserve su protagonismo por un largo tiempo (Alemu et al., 2012). Las nuevas piezas del puzle son: 


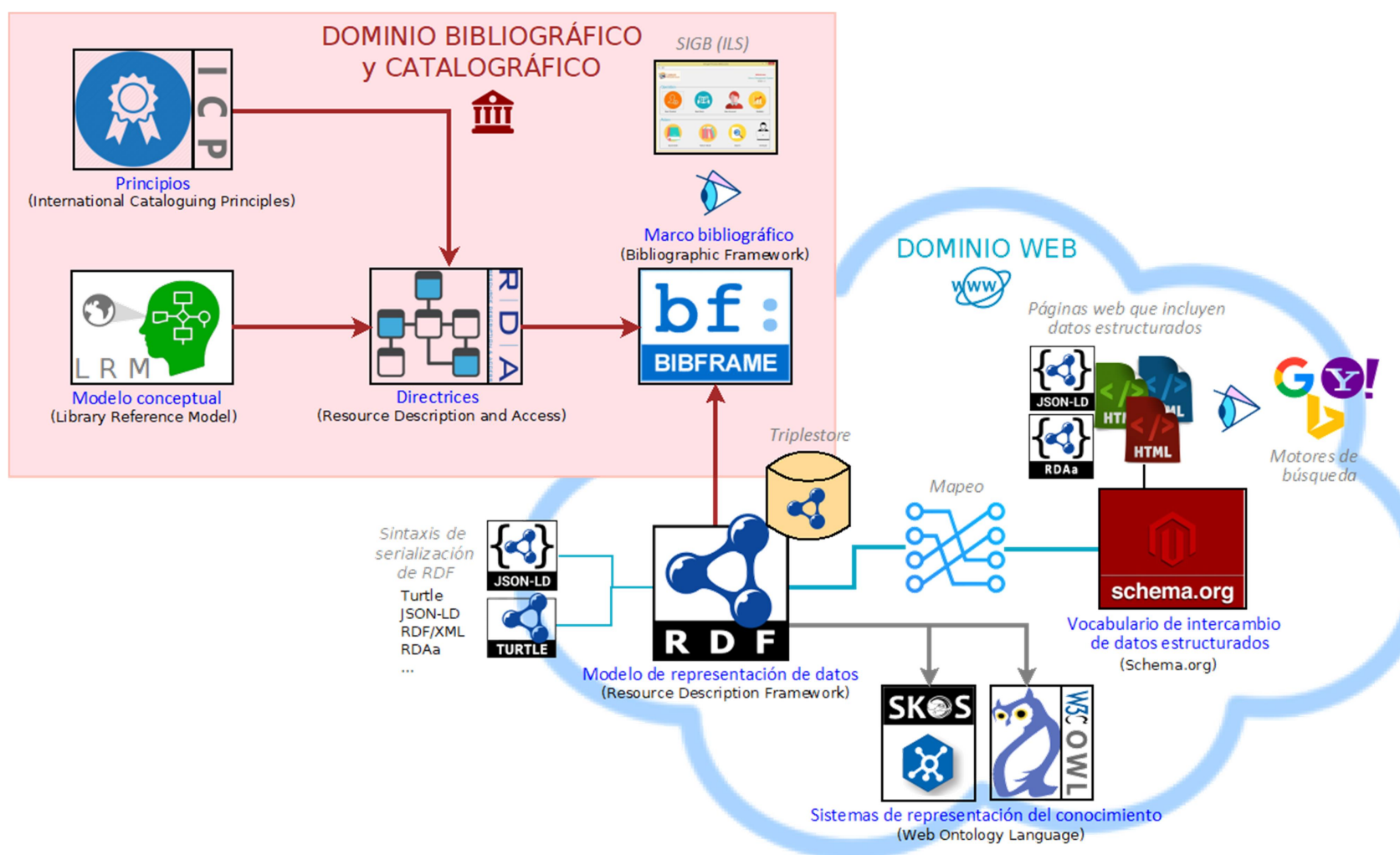

Figura 3. Los elementos que integran el nuevo contexto bibliográfico en las bibliotecas y sus relaciones. Elaboración propia.

ICP (International Cataloging Principes), de la IFLA (International Federation of Library Associations and Institutions), recoge principios, objetivos y pautas destinados a servir de orientación para el desarrollo de las normativas de catalogación y las decisiones que han de tomar los catalogadores. Sucesoras de los Principios de París, contemplan tanto los datos bibliográficos como los de autoridad, y en definitiva los distintos conjuntos de datos creados por las bibliotecas, intentando ofrecer una aproximación coherente a la catalogación descriptiva y por materias de todo tipo de recurso bibliográfico (Galeffi et al., 2016).

LRM (Library Reference Model), de la IFLA, es un modelo de referencia conceptual de alto nivel para los datos bibliográficos, desarrollado según un marco de modelado de entidad-relación (E-R). Adopta un enfoque consistente en identificar las entidades fundamentales que integran los recursos bibliográficos, sus atributos o características, y las relaciones entre estas que son importantes para la búsqueda de información bibliográfica y la navegación entre sus datos. 


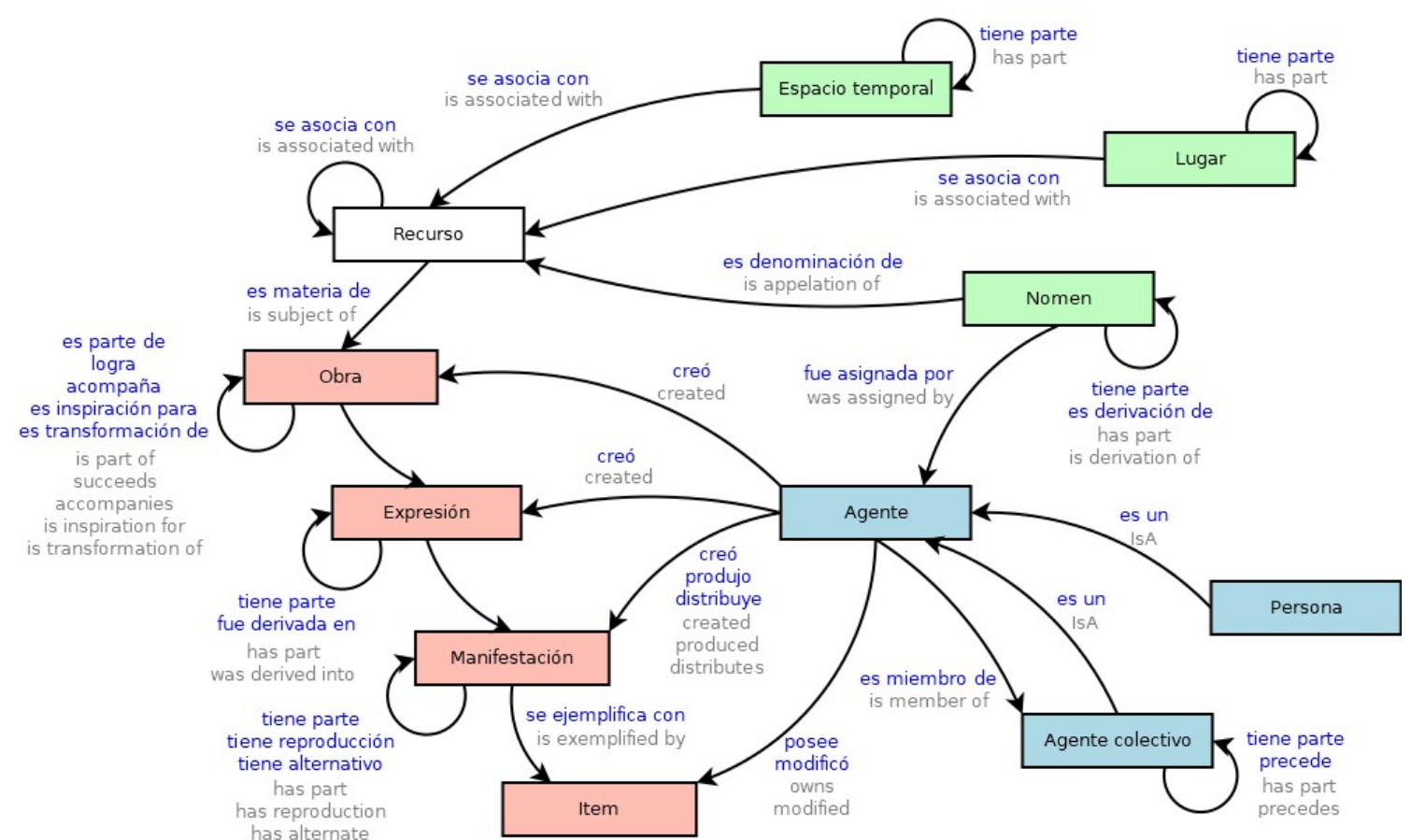

Figura 4. Diagrama de E-R con las relaciones entre entidades del modelo LRM. Traducido de Riva, Le Boeuf y Zumer

(2017).

Pretende servir como guía para la elaboración de reglas de catalogación y la implementación de sistemas de información bibliográficos. El modelo es el resultado de la reciente consolidación de la familia de requisitos funcionales integrada por los modelos FRBR (Functional Requirements for Bibliographic Records), FRAD (Functional Requirements for Authority Data) y FRSAD (Functional Requirements for Subject Authority Data).

RDA (Resource Description and Access), del RSC (RDA Steering Committee), es un conjunto de pautas, directrices e instrucciones para la creación de metadatos de recursos tanto relativos a bibliotecas como referentes al patrimonio cultural, que sean apropiados y estén adecuadamente descritos para su uso en aplicaciones de datos enlazados centradas en el usuario (RDA Steering Committee, 2017). Considerado como relevo de las AACR2, dota de contenido y desarrolla el marco teórico establecido por el modelo de referencia LRM, fundamentado en un conjunto de entidades y sus atributos y relaciones.

Sus especificaciones se publican en línea en el sitio de RDA Toolkit, mantenido por la ALA (American Library Association). Las diferentes secciones cubren atributos y relaciones de entidades que apoyan una tarea específica del usuario, en base a los principios internacionales de catalogación, ICP.

El contenido especificado en RDA es independiente del contenedor, de modo que puede transmitirse mediante cualquier esquema de codificación de metadatos, como MARC 21, DC (Dublin Core), MODS (Metadata Object Description Schema) o BIBFRAME (Bibliographic Framework) (Mering, 2014) de la Library of Congress (LC), sin duda el entorno que mejor implementa sus directrices de catalogación y compartición de recursos bibliográficos. 


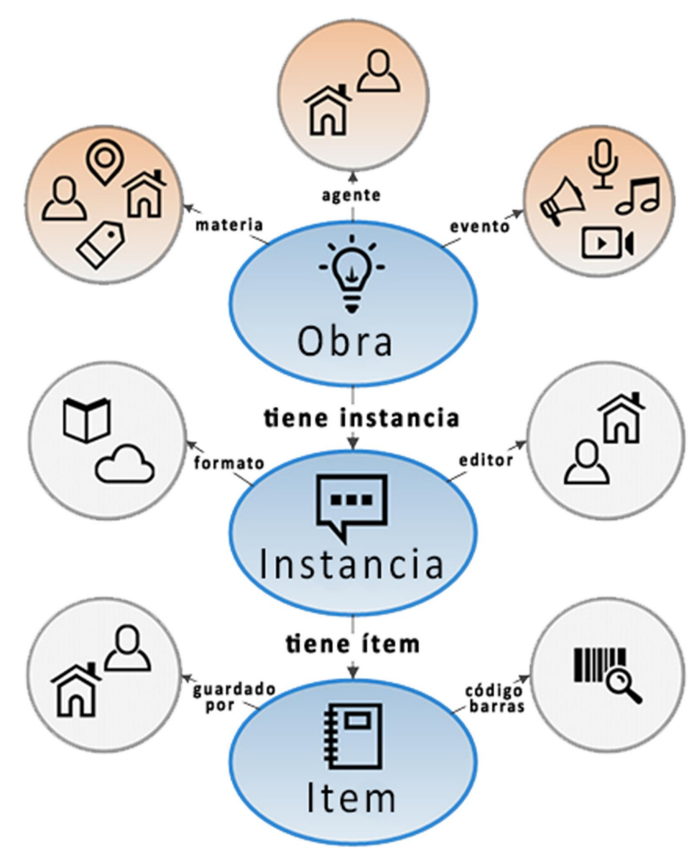

Figura 5.Organización de conceptos en BIBFRAME. Traducido de Library of Congress (2017).

El formato de codificación de metadatos BIBFRAME es un estándar de descripción bibliográfica concebido por la comunidad bibliotecaria, que emplea tecnologías de datos enlazados para sacar el máximo partido a las enormes ventajas ofrecidas por la web semántica y que permite así exponer los datos bibliográficos en la web, traspasando las fronteras de la propia biblioteca (Library of Congress 2017). A pesar de que se concibe para ser agnóstico respecto a las reglas de catalogación, lo cierto es que existe una importante sintonía entre las directrices de RDA y las entidades, atributos y relaciones de su modelo conceptual LRM y las clases y propiedades (correspondientes a características y a relaciones) de BIBFRAME.

El vocabulario se compone de clases y propiedades de RDF (Resource Description Framework), modelo estándar de W3C (World Wide Web Consortium) para el intercambio de datos en la web (RDF Working Group, 2014). Dicho marco extiende la estructura de enlaces de la web mediante el uso de tripletas o sentencias RDF, declaraciones sobre recursos web en forma de expresiones sujeto-predicado-objeto, cada uno de los cuales se identifica a través de una URI (Uniform Resource Identifier).

La consulta y manipulación de datos almacenados en formato RDF (triplestores), se realiza a través del lenguaje SPARQL (acrónimo recursivo de SPARQL Protocol and RDF Query Language). Los puntos de acceso (endpoints) son servicios que aceptan sentencias SPARQL sobre datos RDF a los que se tiene acceso, devolviendo los resultados buscados. 


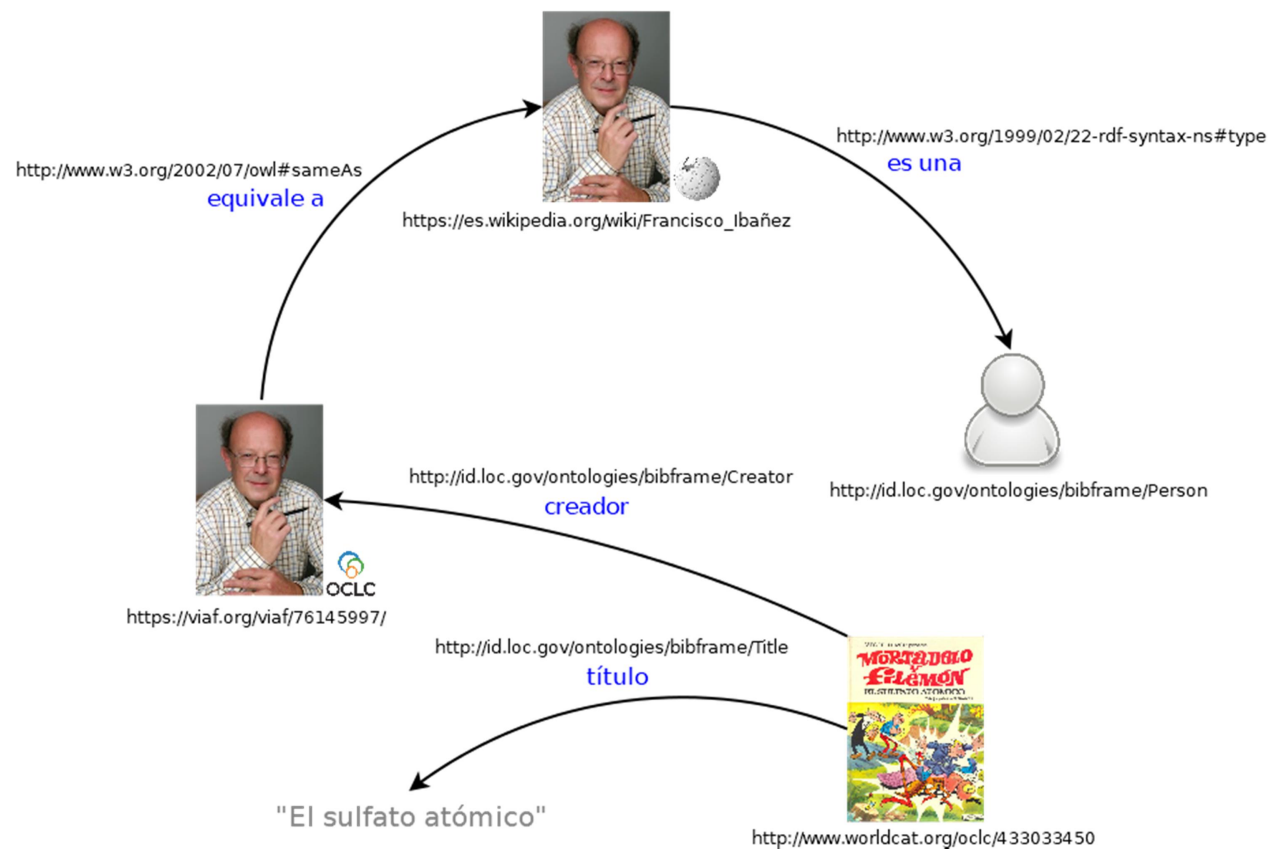

Figura 6. Grafo con tripletas RDF de sujeto, predicado y verbo. Se incluyen posibles URI para cada elemento. Elaboración propia.

Por otra parte, para hacer accesible la información dispuesta en la web a través de los buscadores de internet, esta debería ofrecerse en un formato de datos estructurados marcado por Schema.org, conjunto de vocabularios creados para el intercambio de informes y datos estructurados entre publicadores y consumidores de los mismos en la web (Guha, Brickley y Macbeth, 2015).

Mientras que las tripletas RDF, respondiendo al modelo de BIBFRAME, permiten especificar el contenido de los recursos bibliográficos y de autoridad con gran nivel de detalle y son apropiados para su interacción con los futuros SIGB (Sistemas Integrados de Gestión Bibliotecaria, o en inglés ILS, Integrated Library Systems) de última generación, Schema.org define vocabularios de carácter más general y de diversa tipología en un conjunto de esquemas, con el fin de incrustar datos estructurados en páginas web para su uso en motores de búsqueda y otras aplicaciones de la web.

En efecto, diferentes ontologías pueden servir para distintos propósitos y contextos. Dependiendo del objetivo que una institución tuviera, podría convertir o generar los datos albergados en sus repositorios según uno u otro formato. En todo caso, siempre que el contenido informativo fuera suficiente, es posible transformar la estructura de los datos a través de un apropiado mapeo de ontologías. Por ejemplo, llegado el caso, se podrían traducir las tripletas RDF catalográficas almacenadas en una institución que siguieran el modelo BIBFRAME ( $u$ otro equivalente que alternativamente podría emplearse) en datos estructurados según Schema.org, con la conveniente traducción de vocabulario y propiedades de uno al otro.

\section{METODOLOGÍA Y OBJETIVOS}

\subsection{Preguntas}

Las preguntas fundamentales que se pretende responder a través de este estudio de investigación son:

- ¿Qué ventajas aporta el cambio de paradigma de catalogación a los usuarios y a las bibliotecas, y qué implicaciones tiene en el seno de las mismas y en la propia comunidad bibliotecaria?

- ¿Cuáles son los principales problemas a los que se está enfrentando la comunidad bibliotecaria a la hora de abordar el proceso de transformación hacia las nuevas prácticas de catalogación?

- ¿Qué estrategia debe asumir y qué pasos deben dar las bibliotecas para acometer y consumar el proceso transformador al que se enfrentan?

- ¿Cuál es la situación de la implantación de la nueva filosofía de catalogación, en especial dentro de las fronteras del estado español? 


\subsection{Método de investigación}

Ante un escenario de trabajo de estas características, que aborda diversidad de aspectos bajo distintos prismas (estándares, modelos, buenas prácticas y reglas de catalogación; iniciativas, colaboraciones y pruebas piloto; sistemas integrados de bibliotecas y herramientas de transformación y publicación) y con personas de interés que juegan gran variedad de roles y pertenecen a instituciones de distinta tipología (desarrolladores de estándares, implementadores de software, profesionales de bibliotecas, usuarios de internet...), se ha optado por abordar un enfoque de estudio eminentemente cualitativo.

En primer lugar, se ha buscado contextualizar las actuales reglas de juego -notablemente cambiantes en un contexto tan convulso como el del control bibliográfico- mediante un examen de artículos y conferencias relevantes sobre la materia en la actualidad reciente. $\mathrm{Su}$ contenido ha permitido responder con cierto nivel de profundidad algunas de las preguntas planteadas, y determinar quiénes son los principales protagonistas del movimiento revolucionario catalográfico.

A fin de completar y contrastar la información extraída, y concretar cómo es la realidad actual del ejercicio de la catalogación en España, se ha contactado con varias de estas entidades implicadas dentro del panorama autonómico y nacional, con las que se han entablado conversaciones y realizado entrevistas en profundidad. Las instituciones bibliotecarias elegidas son de variada tipología y juegan diversos papeles en el asunto, buscando así obtener una visión completa sobre el proceso de adaptación de RDA y BIBFRAME en España, con especial énfasis en las iniciativas acometidas por las bibliotecas nacionales del estado (BNE y BC):

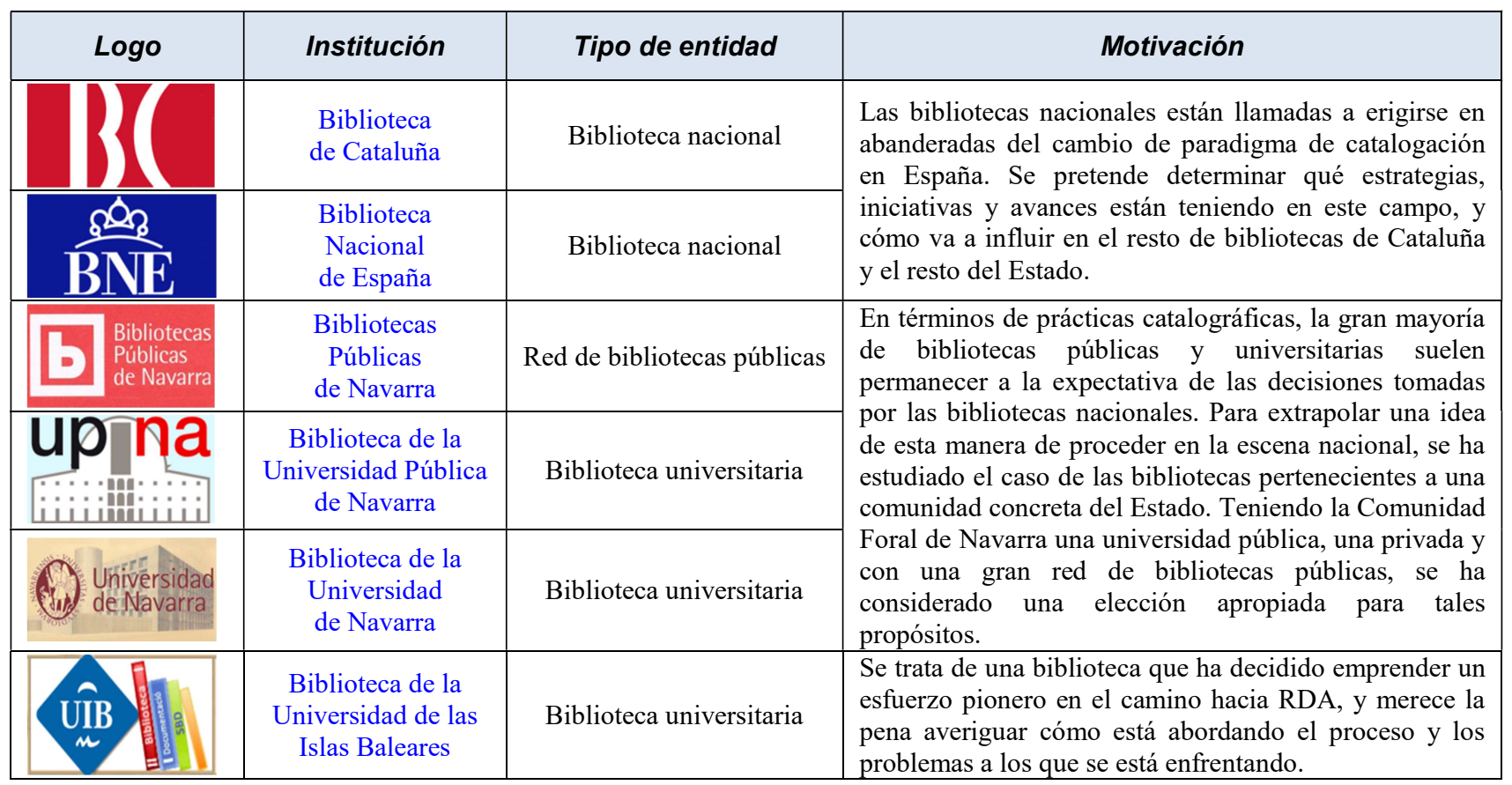

Tabla I. Relación de instituciones y organizaciones con las que se ha contactado.

Las preguntas planteadas a cada una de ellas (véase Anexo) han sido adaptadas al rol protagónico, el ámbito de influencia y el área en el que desempeñan su propia actividad. Toda esta información obtenida ha permitido aclarar en gran medida la situación actual del marco de trabajo y las intenciones futuras para abordar la gestión, el control y la publicación del contenido bibliográfico en las bibliotecas, y precisar las relaciones establecidas entre instituciones internacionales, empresas de software de bibliotecas y bibliotecas nacionales, universitarias y públicas implicadas en la concreción y evolución de esta nueva visión.

\section{RESULTADOS}

\subsection{Bibliotecas de Navarra: a la expectativa de las decisiones de la Biblioteca Nacional de España}

Las principales instituciones bibliotecarias dentro de la comunidad foral son la biblioteca de la Universidad Pública de Navarra (UPNA), la biblioteca de la Universidad de Navarra y la Red de Bibliotecas Públicas de Navarra. En los tres casos permanecen fundamentalmente a la expectativa de los próximos pasos que dé la BNE, que recientemente 
anunció el comienzo de las prácticas de catalogación en RDA para principios de 2019, siempre con MARC 21 como formato de codificación de los registros. Hay que tener en cuenta que gran parte del trabajo de catalogación de los fondos bibliotecarios consiste hoy en día en capturas de registros ofrecidos por la BNE, de modo que -al menos formalmente- RDA formará parte de la estructura de los registros de las bibliotecas, en la medida que la BNE implante estas directrices de catalogación.

Eso no es impedimento para que se haya intentado acometer ciertos avances en la aplicación de criterios de catalogación de RDA, incluyendo la incorporación de algunos campos, ayudadas por el hecho de que sus sistemas de gestión bibliotecaria en la actualidad ya lo permiten (Sierra de Innovative en el caso de la Universidad de Navarra, y AbsysNET en el caso de la UPNA y de la red de bibliotecas públicas). Por ejemplo, se intenta desterrar el uso de abreviaturas en favor a descripciones más completas en distintos campos, y se van añadiendo campos MARC de catalogación RDA para los recursos en línea (campos 336, 337 y 338, referentes a contenido, medio y soporte). Adicionalmente, en el caso de la biblioteca de la UPNA, a la hora de especificar los fondos de los ejemplares, se está tendiendo a considerar cada ejemplar como un fondo nuevo, tal como prescribe RDA.

Hay muy pocos técnicos y encargados de biblioteca formándose en RDA. Asumen esta labor a tiempo parcial y lo hacen mediante cursos sustentados en el RDA Toolkit, algunas prácticas de catalogación y asistencia a jornadas bibliotecarias centradas en esta temática. En el caso de la biblioteca de la UPNA, existe un equipo de estudio sobre RDA se reúne para poner en común los distintos aspectos que constituyen la experiencia catalogadora según estas directrices, amén de participar en iniciativas en este ámbito organizadas por el grupo de bibliotecas universitarias G9 del que forma parte. En todo caso, llegado el momento, se tendrá que abordar la necesidad de constituir grupos de formación, definir las políticas y ámbitos de actuación y adaptar los SIGB para asumir las nuevas directrices catalográficas.

En esta fase temprana, hay disparidad de opiniones entre los catalogadores. Los hay que ven con buenos ojos la llegada de RDA y abogan por el cambio, gracias a las posibilidades que brinda para establecer un mayor nivel de desagregación y detalle en la descripción de los recursos y ejemplares, mientras que otros se cuestionan la necesidad de un cambio de tales proporciones al que se van a enfrentar. A este respecto, hay que tener en cuenta que en el seno de las bibliotecas de la comunidad todavía no se está familiarizado con el marco bibliográfico de BIBFRAME y el papel clave que jugarán en un futuro los datos enlazados en el contexto de la web. Supuestamente esto llegará con el tiempo, cuando haya un avance más significativo en las especificaciones del estándar y en la implementación de las herramientas bibliotecarias necesarias para su uso, a manos de las instituciones de referencia del sector.

\subsection{La Biblioteca de la UIB: pioneros de la implantación de RDA en España}

Sin lugar a dudas, la de la UIB es una de las bibliotecas universitarias que más iniciativas está tomando para hacer un seguimiento y generar contenido formativo sobre las nuevas directrices de catalogación. En concreto, el servicio de Biblioteca y Documentación tiene un área del sitio web de la biblioteca reservada a información sobre catalogación en RDA, que incluye una serie de videos y documentos breves sobre las principales novedades de la catalogación en RDA frente a las reglas de catalogación previas (ISBD y AACR2), así como documentación de cómo ha sido el proceso de implementación de RDA en la biblioteca.

El trabajo realizado por la biblioteca de la UIB y el material de formación que han confeccionado ha suscitado el interés de la BNE y en especial de la BC. Su MOOC (Massive Open Online Course) sobre catalogación con RDA en la plataforma MiriadaX, cuya primera edición se impartió en marzo de 2017, ha gozado de gran seguimiento por parte de los profesionales de bibliotecas de todos los lugares España.

En noviembre de 2014 comenzó el proceso de implementación de las nuevas instrucciones de catalogación de RDA en la biblioteca, en detrimento de las AACR2. Se hizo sobre MARC 21 y sopesando los pros y los contras (UIB, 2016). El desarrollo del proceso de implementación constó de varias fases, que se iniciaron tras la creación del grupo de trabajo sobre RDA en el seno de la biblioteca de la UIB:

1. Cambio de mentalidad ante la catalogación en RDA (2014-2015). Se procedió a la redacción de manuales y guías sobre las entidades y relaciones, y sobre la evolución de los campos MARC desde AACR2 a RDA.

2. Relación de datos y enriquecimiento de registros (2016-2017). Se inició un proyecto de adaptación de los registros de autoridad a las nuevas directrices de catalogación, y comenzaron los cambios de los registros retrospectivos pre-RDA.

3. Mejora de visualización y recuperación (2017-2018). Se ha revisado y sacado conclusiones de los resultados de la fase anterior, y se está trabajando en el análisis de cómo acometer el proceso de migración de los registros exis- 
tentes y cómo adaptar la visualización de los registros y las búsquedas, filtros y facetas para hacerse eco de los cambios sobrevenidos en el contenido.

El SIGB tuvo que evolucionar para reflejar estos cambios en el contenido de las especificaciones de catalogación. En la UIB se utiliza Sierra de Innovative, que por otra parte es un software supuestamente preparado para dar respuesta al nuevo estándar. La necesidad de incorporar al catálogo un cada vez mayor número de registros híbridos (físicos y electrónicos) ha sido uno de los detonantes que han llevado a la biblioteca de la UIB a apostar por comenzar a catalogar con RDA en una fase tan temprana.

Uno de los principales retos ha sido la formación que los catalogadores han recibido, previa a abordar la implantación definitiva de RDA. Ha sido necesario que adquieran los conocimientos y habilidades para catalogar según las nuevas directrices, teniendo en cuenta además que el sistema de gestión bibliotecaria no ofrece etiquetas descriptivas apropiadas para ciertos campos MARC, permite además cometer determinados errores en el proceso de catalogación, o bien no aclara la causa de los mismos, algo que el profesional debe contemplar.

Aún así, tras la formación y las primeras experiencias, los catalogadores de la biblioteca consideran que los modelos conceptuales sobre los que se asientan las instrucciones de RDA aportan lógica y consistencia al proceso, y se muestran favorables al cambio. En el proceso de catalogación se incorpora todo tipo de relaciones, incluidas las específicas de FRBR-LRM entre obras, expresiones, manifestaciones, entidades o personas. Esto genera debate sobre qué entidades es necesario crear y relacionar, y en última instancia enriquece el contenido de lo catalogado. No obstante, la continuación de este depende ya del grado de madurez y las condiciones de uso del nuevo sistema BIBFRAME, a cuyo formato presumiblemente se migrará desde MARC 21.

RDA deja mucho margen de criterio a las instituciones para que establezcan sus propias pautas de catalogación, lo que ha obligado a concretar el perfil catalogador en la biblioteca, algo que se ha ido haciendo progresivamente, conforme se iba aprendiendo gracias a la experiencia ganada. La creación del manual de catalogación ha permitido adicionalmente reducir el número de licencias necesarias del RDA Toolkit, con el consiguiente ahorro de costes.

El proceso de migración es un gran desafío al que se enfrentan en la actualidad. Obviamente, es importante intentar automatizar lo más posible la transformación en bloque de los registros retrospectivos en AACR2 en RDA según las especificaciones establecidas. Sin embargo, no siempre es evidente este proceso, dependiendo del tipo de recurso y la información almacenada (por ejemplo, para dirimir si un recurso es texto u ordenador). También se producen importantes dificultades a la hora de establecer equivalencias entre ciertos campos MARC. Por regla general se intenta transformar el contenido existente, sin generar nuevos enlaces o información adicional que permita RDA, al ser una gran carga de trabajo.

Es en colecciones en las que hay un gran interés de que contengan una gran riqueza informativa y que se vea incrementada su visibilidad -como en la biblioteca levantina- en las que se hace un esfuerzo extra de enriquecimiento de los nuevos registros en RDA. Presumiblemente, con la llegada de BIBFRAME y las herramientas de migración que está desarrollando la Library of Congress, este proceso de migración se verá mejorado. También ayudará a que el usuario de la biblioteca perciba realmente en el OPAC (Online Public Access Catalog) una mejora patente en las prestaciones, algo que actualmente no ocurre.

A pesar de la gran convicción con que el equipo del servicio de Biblioteca y Documentación de la UIB está abordando todos los retos, hay que reconocer que la llegada del proyecto 3R (RDA Toolkit Restructure and Redesign Project) supone un pequeño escollo en el camino de la evolución hacia RDA que están recorriendo, e implica asumir nuevos cambios en el proceso de catalogación. El principal motor para ellos es que el usuario vea el trabajo reflejado, y efectivamente se vea beneficiado por este esfuerzo.

\subsection{Las bibliotecas nacionales: referentes de RDA en el Estado}

La BNE y la BC diseñan de forma independiente su propia hoja de ruta y estrategia de implementación, aunque su relación es bastante estrecha y se apoyan y consultan mutua y constantemente.

\subsection{La BC: de las AACR2 a RDA}

La implantación de RDA en la BC se ha desarrollado por fases (Ferran, 2017):

- La primera, iniciada en 2014, fue de decisiones sobre la adopción RDA como evolución natural de las reglas de catalogación AACR2 tras el anuncio de la LC de asumir esta estrategia en detrimento de unas posibles AACR3.

- La segunda fue la fase de difusión del cambio a través de blogs, boletines, actos, jornadas de documentación y entrevistas. Se habilitó un espacio web donde se ofreció a la comunidad documentación técnica confeccionada. 
También se sopesó cómo debería ser la visualización de la información en el OPAC y se implementaron ejemplos para el catalogador.

- La tercera fase fue de pre-implementación. Se concretó la forma de preparar los catálogos, los cambios en los registros, la convivencia de registros pre-RDA y RDA, y los manuales de formación, guías para los catalogadores y documentación técnica. Se progresa en la confección de unas políticas de catalogación propias de la institución, y se afronta la formación en cascada con la impartición de cursos teóricos y prácticos, de una semana de duración para cada tipo de registro.

- La fase cuarta es la de implementación, que se inició a comienzos de 2016 en la propia BC y que se está extendiendo en 2017 y 2018 al resto de bibliotecas universitarias y públicas de Cataluña. La BC ejerce una labor de revisión de las obras, tanto a nivel colectivo como institución por institución.

- La quinta es la fase de post-implementación, donde se concertarán reuniones, se elaborarán estudios de cómo evoluciona la adaptación, se emprenderá el proyecto de publicación de datos enlazados y se abordarán los cambios en catalogación motivados por el proyecto 3R de la LC.

La documentación facilitada por la LC ha sido de gran utilidad, y el concretar un grupo líder para marcar los pasos y establecer vínculos entre instituciones catalanas ha sido un acierto. El proceso ha sido largo y gradual, y todavía no ha finalizado, pero no ha sido traumático. Cada institución ha podido seguir su ritmo. Asimismo, la página creada consagrada al nuevo estándar de catalogación, "Hacia RDA", está siendo muy visitada, y sirve de apoyo y guía a las bibliotecas catalanas.

La evolución hacia RDA no ha cambiado grandemente la relación entre las distintas instituciones bibliotecarias catalanas. Es una relación de igual a igual, con roles divididos. El rol de la BC es principalmente normativo y difusor. Existen tres grandes catálogos en Cataluña: uno universitario y dos públicos (el de la Diputación y el de la Generalitat). Sí que se han creado una serie de comisiones para la dinamización del proceso. Cataluña es una comunidad relativamente pequeña. Por eso el diálogo es más sencillo y ágil que en España, donde la BNE tiene que comunicarse con un gran número de bibliotecas de las distintas comunidades autónomas.

Sin embargo, para el uso de RDA hay muchas prácticas abiertas a los propios criterios de las instituciones. Esto da mucha flexibilidad, pero tiene el inconveniente de que hay que preparar y revisar qué está permitido y qué no, y realizar un seguimiento de la actividad los catalogadores, por estar sujeta a muchas interpretaciones. Se eliminan las reglas restrictivas propias de AACR2, y es necesario elaborar manuales y guías que aclaren qué hacer frente a conflictos en la catalogación.

Se ha observado un incremento de la calidad de catalogación, pero para concretarlo es necesario tener un profundo conocimiento de FRBR-LRM. Respecto al uso de RDA Toolkit, los catalogadores necesitan un proceso de adaptación a la estructura, y queda pendiente un curso de formación acerca de su uso. En contrapartida, la productividad en la generación de registros se ha reducido en un $25 \%$. Conforme se va adquiriendo experiencia, este desfase se va reduciendo, aunque todavía es sensible.

El paso de AACR2 a RDA ha implicado algunos cambios conceptuales en la confección de los registros y el establecimiento de relaciones, destacando el caso de las compilaciones, donde se tiene en cuenta lo marcado por el modelo FRBR-LRM, aunque con las limitaciones que plantea el formato MARC, que sigue siendo el utilizado. Hay que tener en cuenta que BIBFRAME es todavía un formato en ciernes y en proceso de evolución. Además, la LC está trabajando en herramientas y tablas de transformación de BIBFRAME a MARC 21, que no están perfeccionadas y exigen un gran esfuerzo de cooperación entre instituciones, de forma que no se puede realizar una traducción automática de uno a otro formato, ni extraer de forma evidente sus relaciones.

Por eso, el proceso de catalogación sigue siendo similar, habiendo fundamentalmente cambios formales. Nuevos campos y subcampos específicos de las directrices RDA y evitar el uso de abreviaturas son dos de los más destacados. Se siguen utilizando los mismos SIGB (en la BC utilizan Sierra de Innovative), que han evolucionado para dar soporte a las nuevas especificaciones. El hecho de que tanto para AACR2 como para RDA se utilice hoy en día MARC como formato de codificación ha permitido esta circunstancia continuista. Circunstancia que ha hecho que registros preRDA y RDA puedan convivir, y que la migración no sea un problema perentorio.

Las primeras labores de transformación se centran en los datos de autoridad y después se afrontará una labor de "maquillaje" de los registros bibliográficos, en la que se determinarán cambios mínimos necesarios a realizar para pasar de AACR2 a RDA, sin imponer la creación de información adicional permitida por las nuevas directrices. Cabe destacar que la Universidad de Barcelona (UB) sí que se plantea la recatalogación completa de sus registros, dando lugar a una mayor riqueza de contenido de su catálogo. 
Existe un proyecto pendiente para la publicación de la valiosa información que poseen los catálogos de las bibliotecas de Cataluña en forma de datos abiertos enlazados (Linked Open Data), en los que cada recurso tiene asociado una URI (Uniform Resource Identifier) que lo identifica unívocamente y lo hace susceptible de ser compartido en la web. El nivel de ambición del proyecto dependerá de la cantidad de recursos que disponga la Generalitat. Existe colaboración con el Open Metadata Registry para el aprovisionamiento de servicios a desarrolladores, y también una publicación de datos de autoridad en VIAF (Virtual International Authority File), pero también se pretende que el catálogo de autoridades de la BC -CANTIC- se exponga y sea reconocido en la web semántica.

\subsection{La BNE: de las RC españolas a RDA}

El punto de partida son las Reglas de Catalogación españolas, basadas en ISBD para la catalogación, CDU (Clasificación Decimal Universal) para la clasificación y los Encabezamientos de Materia de la BNE para la indización, codificado todo ello en MARC 21. En 2014 se valoró la posibilidad de comenzar con el proceso de evolución hacia RDA, pero no fue hasta 2015, cuando, tras cierto trabajo previo, se vio una situación propicia para ponerse manos a la obra. En todo caso, se formó un grupo de trabajo de RDA, que empezó a examinar los documentos y estudios existentes sobre el tema, y se realizó un análisis de las entidades y relaciones (esquema o modelo conceptual) que se planteaban.

La llegada del nuevo estándar debería aportar ventajas, como una integración del contenido bibliotecario a la web semántica o la oportunidad para unificar las prácticas bibliotecarias a nivel nacional, pero también desventajas, siendo los costes de licencias, la implementación y la productividad las principales, así como las implicaciones de la convivencia de registros antiguos y nuevos en su catálogo.

El proceso de implementación de RDA va a ser gradual, desde enero de 2019 hasta junio de 2020 (Santos, 2017). Se iniciará con la modificación de los puntos de acceso (los registros de autoridad). Se procurará que sea un proceso lo más automatizado posible, pero no se podrá evitar realizar actividad manual y se incrementará el nivel de codificación. Se continuará con la creación de registros bibliográficos correspondientes a monografías modernas impresas y después se seguirá con el resto de materiales. Puede haber cambios en el orden de implementación de los distintos materiales.

La codificación sigue siendo en MARC 21, y la estructura de los registros y puntuación la marcada por ISBD. Se ha ido allanando parte del camino, y actualmente ya se usa de forma extensiva términos o designadores de relación y términos de función, de momento para las relaciones entre recursos y personas, y no todavía de recursos bibliográficos entre sí. Es en las nuevas posibilidades de relaciones que permite el modelo conceptual de FRBR-LRM donde la situación se pone peliaguda. En la estructura de la base de datos de catalogación no está explícitamente reflejada información de estas, de manera que es difícil plasmarlo en los nuevos registros o los registros migrados manteniendo dicho repositorio, como es el caso.

Se puede afirmar que, aparte de la evolución de los campos MARC 21, la forma de introducir la información de registros en RDA no ha supuesto un gran cambio de filosofía. Y es que todavía no hay disponible en el mercado una nueva generación de sistemas de gestión de bibliotecas que incorporen editores de catalogación orientados al modelo FRBR-LRM. En todo caso, se intentará que esa mayor capacidad de desagregación y detalle que permite RDA tenga su repercusión en el OPAC ofrecido a los usuarios de la biblioteca.

En cuestiones de migración de datos, el SIGB de la BNE (Symphony de SirsiDynix) permite gran flexibilidad a la hora de afrontar modificaciones de incorporar nuevos campos en los registros catalográficos de forma masiva, sin necesidad de recurrir a herramientas adicionales. Indudablemente, el límite lo marca el propio sistema de información y el repositorio que lo sustenta. En la actualidad no se ve en los catálogos muchas relaciones entre obras u otras expresiones en las instituciones, y tampoco va a ser el caso en una primera instancia de la evolución hacia RDA.

En la actualidad se está trabajando en el perfil BNE de RDA, relacionado con los flujos de trabajo (workflow) de la biblioteca, es decir, los tipos de descripción, la forma de transcripción de los datos, los elementos núcleo o la aplicación de alternativas. Uno de los principales problemas es que parece que la RDA va en una dirección, mientras que los catálogos trabajan en una dirección distinta. Este entorno -que no es propicio en cuanto a herramientasdificulta el trabajo de implementación de RDA.

Recientemente el RSC ha comunicado a las instituciones que están acometiendo el cambio hacia RDA -entre ellas la BNE, miembro de EURIG (European RDA Interest Group)- que paren temporalmente el proceso de migración 
hacia RDA, en espera de la llegada de grandes cambios en cuanto a las especificaciones de las directrices, auspiciadas por el proyecto 3R. Esta circunstancia puede producir desviaciones en el cronograma previsto.

Por otra parte, se ha creado un espacio web que pretende ser el canal de comunicación con la comunidad bibliotecaria de todos los trabajos que se vayan haciendo sobre los nuevos estándares de catalogación. En todo caso, la BNE prefiere esperar para generar material de formación propio a que haya más estabilidad en las distintas especificaciones para la catalogación, para evitar reciclajes prematuros.

En relación con BIBFRAME, no hay que pasar por alto el hecho de que el RSC lamenta que la LC no tenga más en cuenta su opinión a la hora de diseñar el entorno. En el futuro los catalogadores verán y editarán datos catalográficos según las entidades y relaciones planteadas por el modelo conceptual de RDA, mientras que el contenedor consistirá en las clases y atributos propios de BIBFRAME. Es decir, BIBFRAME se quedaría en los entresijos de la infraestructura, y el catalogador no debería tener necesidad de conocer a fondo sus especificaciones, y sí las directrices de RDA.

El proyecto de web semánticadatos.bne.es es un catálogo en la nube basado en datos enlazados. Las páginas que ofrece se componen de entidades relacionadas (obras, personas, y otras difíciles de ajustar al modelo prescrito) que han podido inferirse de forma más o menos automatizada a partir de los datos legados en MARC 21 de los catálogos de la biblioteca. Como ontología, la BNE asumió una propia, basada en FRBR-LRM, y frente a BIBFRAME, cuya especificación está experimentando convulsos cambios hoy en día, parecen inclinados a tender hacia la descrita por RDA Registry, y estudiar y establecer una correspondencia con sus entidades y atributos. En un futuro próximo se quiere poder sincronizar la información de los registros antiguos con los de la plataforma de datos enlazados, y evitar tener que realizar el trabajo dos veces, que es lo que ocurre en la actualidad. La creación de la plataforma de datos abiertos es casi como nadar contracorriente, porque las herramientas actuales (entre ellas los editores de catalogación) parecen seguir todavía una filosofía diametralmente opuesta a la que proponen los datos enlazados.

El perfil del catalogador va a cambiar indudablemente. Se requiere un mayor conocimiento de los aspectos informáticos y una relación más estrecha con el departamento tecnológico. La necesidad de tratar y desplegar datos enlazados así lo exige. Los propios responsables de la implantación de RDA han tenido que formarse en cuestiones como RDF para poder adquirir el criterio suficiente para tomar determinadas decisiones sobre la plataforma de datos enlazados.

Posiblemente el núcleo central de la catalogación efectuada por los profesionales en las bibliotecas se concentre en un futuro en el establecimiento de las relaciones y en la asignación de materias y la clasificación. Gracias al esencial proceso de automatización que va a experimentar la catalogación en el futuro desde diferentes fuentes (editoriales, librerías...), el resto del proceso técnico sería menos relevante. En última instancia, el propósito seguirá siendo que la información de catalogación sea accesible en la web en forma de datos enlazados, a disposición de los usuarios de internet.

\section{ANÁLISIS}

Hace mucho que lleva diciéndose que MARC debe morir (Tennant, 2002), y llegará un momento en que MARC morirá, pero de momento goza de buena salud y sigue contemplándose su evolución, fruto de su rol protagónico en estas primeras etapas de evolución del paradigma catalográfico (McCallum, 2017). Procede ir dando pequeños pasos que representen un avance sostenido para finalmente llegar a la meta que supone la definitiva instauración de los nuevos estándares y el despliegue y consolidación de sistemas y herramientas que cumplan con estos. Aún en medio de la vorágine de nuevos modelos, directrices, pautas, marcos de trabajo, y lenguajes de descripción y modelado, y su casi sistemática evolución hacia nuevas versiones, estos pasos son trágicamente necesarios, porque:

- El matrimonio de las actuales reglas de catalogación con el formato MARC da como resultado tablas de contenido catalográfico estructuradas en cabeceras, campos, etiquetas y códigos de subcampo. Y esta larga cadena de información que representa cada registro bibliográfico no es adecuada para su integración en la web, piedra angular de la actual sociedad del conocimiento, principalmente porque:

- Requiere para su interpretación y difusión de sistemas de gestión y aplicaciones propias del ámbito bibliotecario. Esto implica que buscadores y otros portales, a fin de localizar información en los registros de los catálogos, dependen de la funcionalidad ofrecida por la gran diversidad de SIGB desplegados en las distintas instituciones bibliotecarias, y a la postre, de su capacidad para publicar sus datos en la web. Habida cuenta de la heterogeneidad de sistemas de gestión bibliotecaria existente y de las tradicionales limitaciones que muchos de ellos presentan, esto es un gran hándicap. 
- Es difícil establecer relaciones entre entidades bibliográficas y de autoridad en base a estas reglas y formatos. No solo es que no existan prácticamente campos y códigos en las especificaciones de MARC bibliográfico que permitan indicar tales relaciones, sino que estas se harían a una cadena descriptora del recurso en vez de unívocamente (por ejemplo, a través de una URI) a una entidad que contuviese toda la información del mismo.

- Además, RDA, al implementar el modelo LRM, permite desagregar la información más que las antiguas reglas de catalogación, pudiéndose separar de una forma más evidente en correspondientes registros individuales distintas manifestaciones de una misma obra (por ejemplo una física y una electrónica), o distintos componentes de una compilación, y establecer relaciones entre ellos.

- Hay contenido de ciertos campos difícil de desmenuzar con un nivel de detalle suficiente. Por ejemplo, en las actuales especificaciones del formato MARC para registros bibliográficos, el código de subcampo \$c no repetible del campo 245, correspondiente a la mención de título, puede albergar gran diversidad de autores y responsables con distintas funciones, siendo complejo extraer cada nombre y función de manera individualizada.

- Puede haber información duplicada e inconsistente diseminada en distintos campos de un registro bibliográfico en formato MARC. Por ejemplo, tanto el campo 440 como el 490 hacen mención a la serie; o el contenido de las posiciones 35/37 del campo de control 008, referente al idioma del documento, debe coincidir con el albergado por subcampo \$a del campo 041, correspondiente al código de lengua, si este estuviese incluido. La gran mayoría de SIGB no validan la congruencia de este contenido, dando pie a posibles inconsistencias en la información catalográfica.

Como se ha mencionado, son cambios que no se deben posponer a pesar de que los estándares y directrices que integran el nuevo paradigma de catalogación sigan evolucionando, ya que:

- Los usuarios tienen expectativas. Van a Google y otros motores de búsqueda de la web para encontrar lo que buscan, y dejan de lado aquello que no campea a sus anchas por la web. Las bibliotecas no pueden esperar para exponer de forma nativa en la web la valiosa información que atesoran, fruto del trabajo de profesionales muy cualificados sobre la información y la documentación. De renunciar a hacer este esfuerzo, su protagonismo queda en entredicho.

- Es preciso aprender de la propia experiencia, y brindar soluciones a la comunidad y recibir su opinión y punto de vista. Las bibliotecas tienen que ponerse coger el toro por los cuernos e iterar sus desarrollos, sin esperar a que las aguas se calmen y los estándares tomen forma definitiva (Pesch y Miller, 2016).

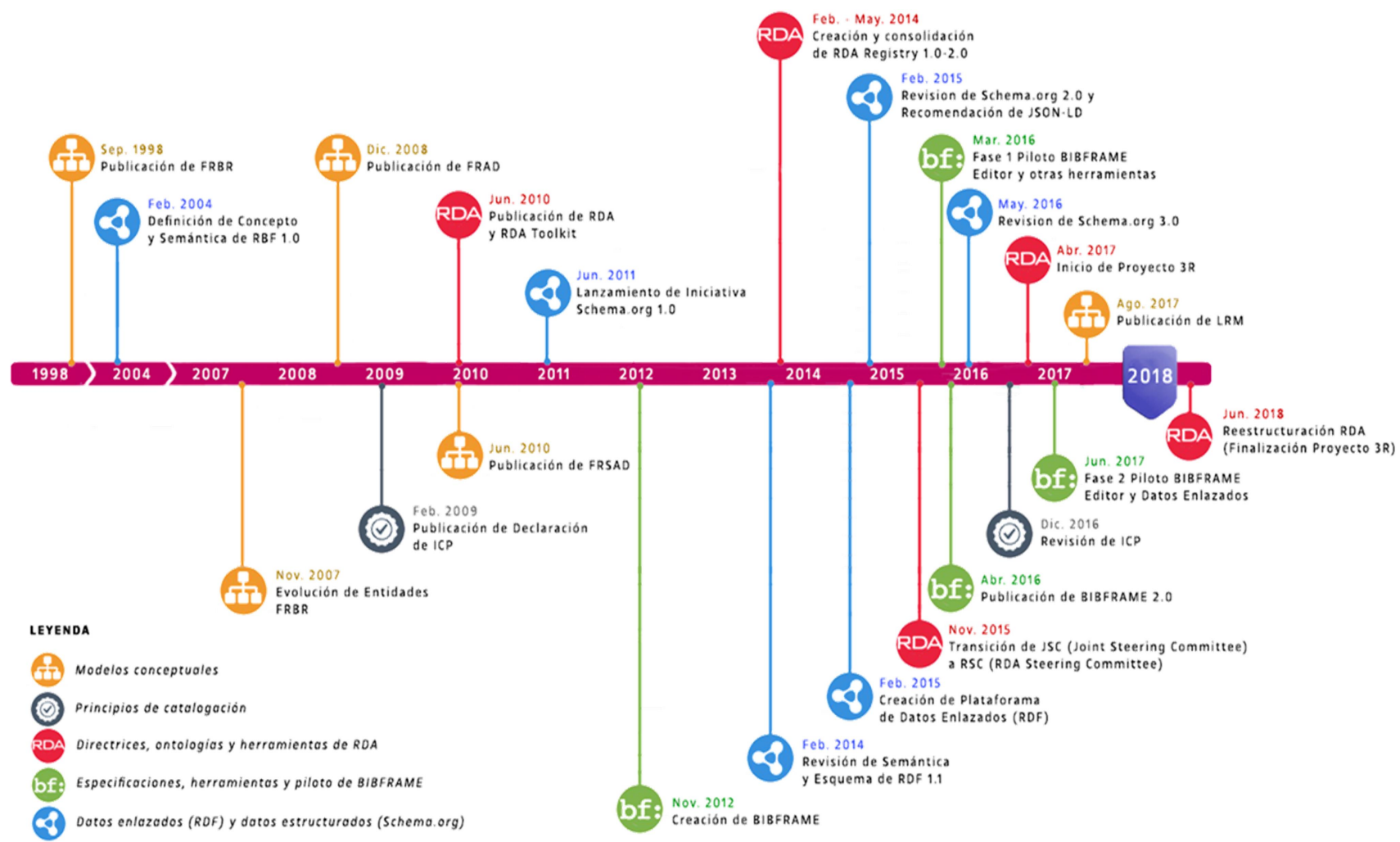

Figura 7. Línea temporal con hitos que afectan a los estándares del nuevo paradigma de catalogación. Elaboración propia. 
Las bibliotecas ya se han puesto manos a la obra, aun y cuando esto implica tener que bailar con RDA en el salón más modesto (MARC) antes de hacerlo en el principal y más elegante (BIBFRAME). Por lo menos unos cuantos bailes, a pesar de que el suelo esté resbaladizo y sea irregular. Y es que el salón preferente está aún en obras lo suficientemente serias como para que todavía no sea posible celebrar fiestas oficiales sobre su parqué.

Así pues, un nuevo discurso catalogador está en plena ebullición, a la luz de la gran cantidad de cambios que se han ido produciendo en sus partes integrantes a lo largo de los últimos años. Es importante entender que el propósito que motiva el cambio no es competir contra la web, sino integrarse con ella. Y por tanto, no se trata de luchar con los buscadores, sino ser visibles para ellos.

La información disponible no tiene por qué ser perfecta, ni los estándares de catalogación y de datos enlazados los definitivos. Es un mundo convulso en el que hay que saber desenvolverse, para ofrecer al usuario lo que necesita y estar a la altura de sus expectativas, creadas como consecuencia de las excelencias en la búsqueda y obtención de resultados informativos que internet brinda y en las que la comunidad bibliotecaria no participa lo suficiente. Se trata de iterar para evolucionar (Miller, 2015).

\subsection{Implementación de RDA con MARC}

Es el primer paso dado por las bibliotecas. Se mantiene el contenedor (formato MARC) y se cambia el contenido (directrices de catalogación RDA en vez de las RC españolas, las AACR2 o la ISBD). Esto es posible gracias al hecho de que las instrucciones de RDA están pensadas para ser independientes del formato o sistema utilizado para el almacenamiento e intercambio y visualización de los datos.

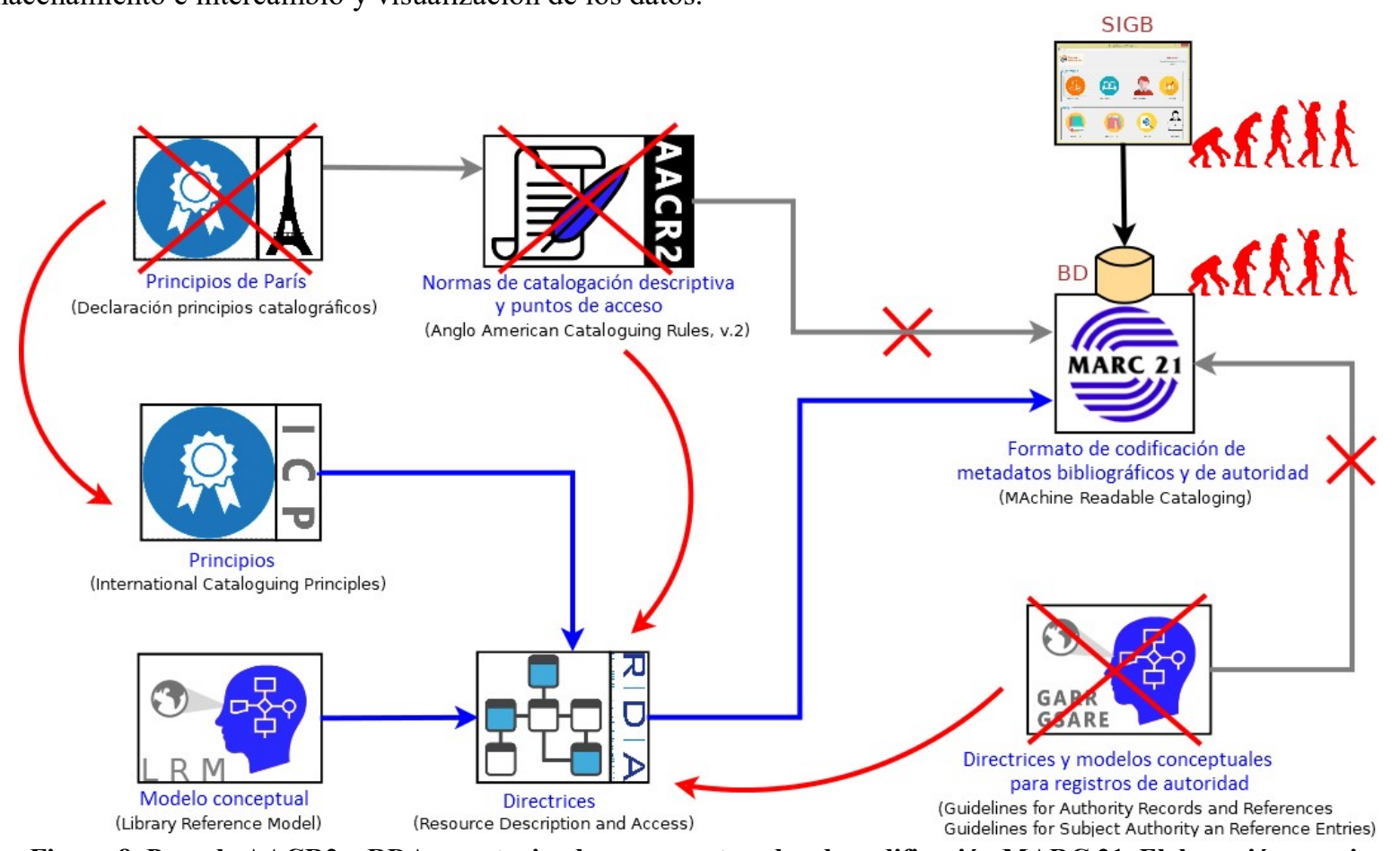

Figura 8. Paso de AACR2 a RDA, manteniendo como contenedor de codificación MARC 21. Elaboración propia.

La evolución del contenido implica una adaptación del formato MARC al nuevo código de catalogación, que incluye la creación de nuevos campos y la redefinición de otros que ya existían. Los datos residen en la misma base de datos, y los sistemas de información bibliotecarios se mantienen, simplificando el proceso de migración, la convivencia de registros RDA y pre-RDA y la continuidad de los SIGB.

Como las directrices de RDA ofrecen gran número de opciones y asignación de contenido que dejan a criterio del catalogador, es necesario que ya en esta primera incursión en RDA las instituciones definan su propio perfil de RDA, en el que se concreten estos criterios en el proceso de catalogación para los distintos tipos de materiales.

La biblioteca de la UIB ha sido una de las primeras en dar este paso, que también están dando de forma escalonada las bibliotecas universitarias y públicas catalanas, al abrigo de la $\mathrm{BC}$, que ejerce de órgano normativo y difusor. Efectivamente, la experiencia está siendo prolongada pero no traumática. La BNE lleva bastante tiempo madurando el 
proceso, y comenzará a catalogar en RDA a principios de 2019, a la que seguirán a un ritmo propio las restantes bibliotecas del país. En todo caso, el proyecto 3R, de revisión y restructuración del RDA Toolkit, está obligando a revisar, reproyectar $\mathrm{y}$-posiblemente- retrasar el proceso transformador.

\subsection{Exposición de los datos enlazados a partir de los registros MARC existentes}

Los bibliotecarios tienen que dejar de pensar en términos de registros bibliográficos y comenzar a pensar en términos de datos, y entender cómo estos se integran con la web semántica a través de los datos enlazados y los estándares que abriga (Coyle, 2007). No se puede esperar a la llegada de los nuevos SIGB que gestionen datos catalográficos en formato BIBFRAME de forma nativa, y ya hay bibliotecas que, a pesar de seguir usando MARC como formato de codificación en sus catálogos, apuestan por traducir la información atesorada como datos abiertos enlazados (a la postre, tripletas RDF), para ser expuesta en la web (Tharani, 2015). Empresas desarrolladoras de software y las propias bibliotecas han de planificar el desarrollo de sistemas capaces de producir datos enlazados compartidos, a partir de los registros actualmente disponibles (Sprochi, 2016).

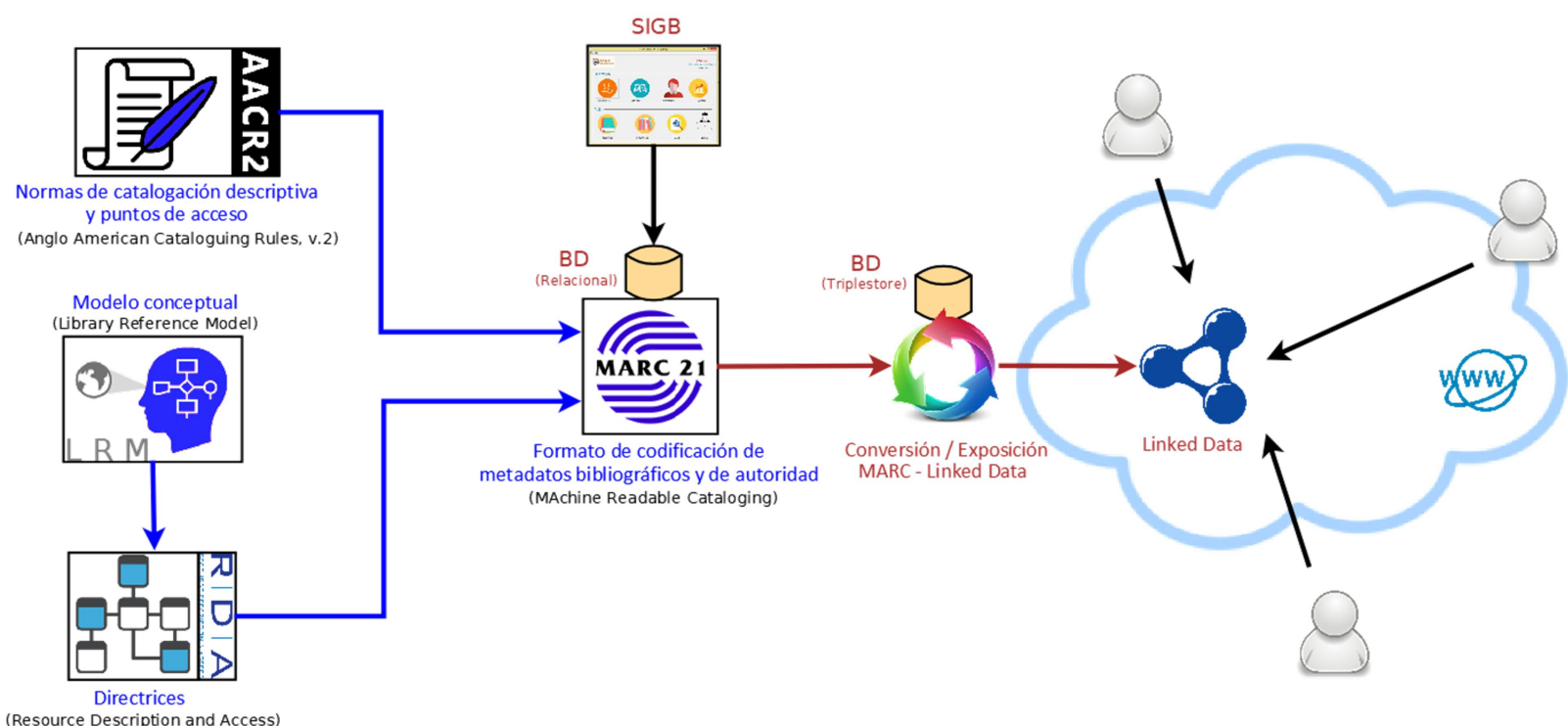

Figura 9. Traducción de registros MARC a conjuntos de datos enlazados (RDF) que se exponen en la web. Elaboración propia.

Al no poder renunciar todavía las entidades bibliotecarias a los registros MARC, y debido tanto a las dificultades que entraña traducir en tiempo real el contenido de estos registros bibliográficos a conjuntos de datos enlazados como al hecho de que mediante la ontología oportuna se puede expresar contenido y relaciones que no es posible plasmar a través del binomio de MARC y las antiguas reglas de catalogación, esto implica el doble esfuerzo de mantener:

- La base de datos relacional con los registros bibliográficos y de autoridad legados.

- Y el repositorio con la información constituida por los datos enlazados, fruto de la traducción y migración de los registros del catálogo a datos accesibles desde la web semántica (tripletas RDF).

Muchas bibliotecas nacionales han realizado proyectos para publicar como datos enlazados sus catálogos y registros de autoridad. En el caso de la BNE, ha desplegado el portal datos.bne.es en forma de un OPAC de consulta, amigable para el usuario. Y es que a la hora de publicar los datos enlazados, aparte de su calidad, es fundamental exponerlos en un sitio o plataforma web que sea fácilmente consultable por el usuario, y no ofrecerlos únicamente para la extracción de información mediante sentencias SPARQL, que los haría solo accesibles para expertos.

El principal problema sobrevenido ha sido el hecho de que las distintas instituciones asumen sus propias elecciones de ontologías e implementan soluciones en muchas ocasiones ad hoc para publicar las tripletas RDF. Tal circunstancia ha dado pie a que, paradójicamente, se pase de tener silos en MARC a silos en datos enlazados (Suominen, 2016). Existe gran número de modelos de datos, ligados a vocabularios y ontologías específicos, tanto dentro del dominio bibliotecario (ISBD, RDA, FRBR, BIBFRAME...) como de autoridades (VIAF), o más generalistas (por ejemplo SKOS (Simple Knowledge Organization System).

En todo caso, en última instancia los datos enlazados publicados en la web son tripletas RDF, los cuales se pueden consultar mediante sentencias SPARQL. Para conseguir relacionar los datos enlazados publicados con otros datos 
enlazados existentes en la web -Europeana, BNE, VIAF, LCSH (Library of Congress Subject Headings)...-, y no pasar de silos en MARC a silos en datos enlazados, la clave es alinear estos estándares de ontologías y vocabularios, es decir, mapear las equivalencias en entidades, relaciones y atributos entre unas y otras ontologías.

La elección del dominio y la ontología, los requisitos de la institución, así como la calidad de los datos originales (en MARC) son los que determinan el proceso de traducción y la calidad de los datos enlazados resultantes, tanto en cuestión de número, como de contenido y de enlaces establecidos entre recursos bibliográficos y autoridades. Idealmente, en aras de una homogeneidad de la publicación de los datos bibliográficos de distintas fuentes que permita una fácil integración entre ellos sin necesidad de mapeos de vocabularios, en el futuro se podría apostar por una ontología común de cara a la publicación de datos enlazados correspondientes a la información bibliográfica albergada por las instituciones. Tras los esfuerzos realizados en este sentido la empresa Zepheira, subcontratada por LC para plantear un proceso de referencia para la publicación como datos enlazados del contenido de los catálogos, primero mediante la iniciativa LibHub y después con Library.Link Network (Ammerman, 2016), esta ontología podría ser BIBFRAME, toda vez que el estándar alcance una mayor madurez.

\subsection{Migración de registros MARC a tripletas RDF de BIBFRAME}

El cambio de contenedor para el contenido RDA, pasando del formato de codificación MARC al modelo de expresión y conexión de datos bibliográficos BIBFRAME es sin duda uno de los pasos más críticos dentro del proceso evolutivo completo. Explicita definitivamente la transición de registros bibliográficos y de autoridad a datos enlazados en la web, e implica pasar de enlazar cadenas (strings) a enlazar cosas (things). La diferencia entre un formato complejo y no adecuado para la web y otro bien diferente basado en vocabularios RDF y un modelo E-R hace que no sea una transformación simple (Pesh y Miller, 2016). Sería ideal empezar de cero, pero no se puede renunciar a recuperar y trasladar todo ese conjunto de metadatos provenientes del pasado almacenados en registros MARC al nuevo estándar BIBFRAME, que los abrirá a la web de una forma más integrada y eficiente (Dull, 2016).

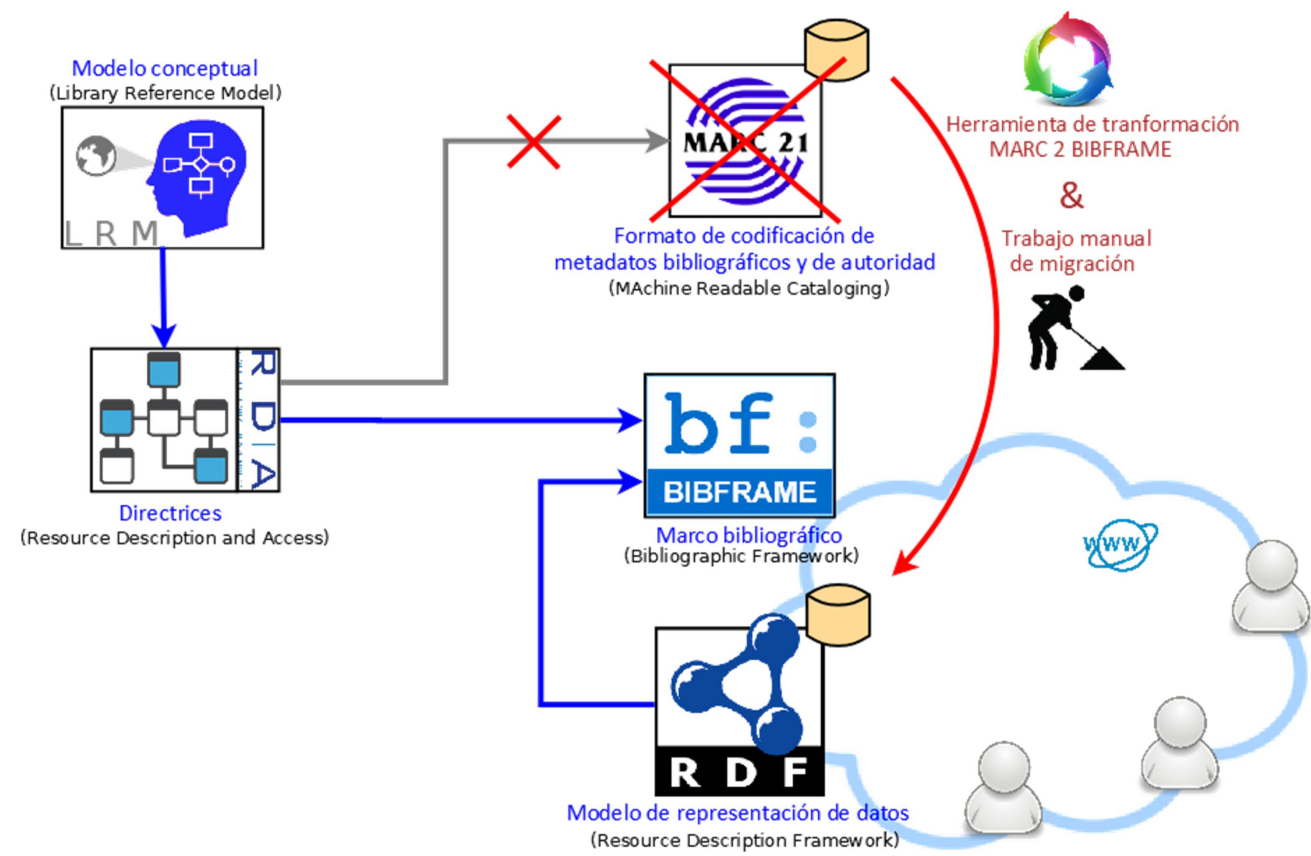

Figura 10. Paso de contenedor MARC 21 a BIBFRAME para RDA. Elaboración propia.

El propósito trae consigo la necesidad de desarrollar herramientas conversoras de MARC a BIBFRAME, capaces de transformar los registros planos en tripletas RDF y extraer las relaciones entre los distintos recursos. Aunque la intención sea conseguir que la mayor parte de este trabajo de migración sea realizado de forma automática, la heterogeneidad de las distintas fuentes de datos, y la mayor riqueza y granularidad de la información en BIBFRAME no garantizan lograrlo de forma íntegra.

La LC ha publicado unas especificaciones para la conversión, así como una herramienta basada en estas marc2bibframe- para convertir registros MARC en datos RDF usando las ontologías BIBFRAME 2.0 y MADSRDF (Metadata Authority Description Schema). Tanto Zepheira como LD4L-Labs (del proyecto Linked Data for Libraries) han emprendido también sus propias iniciativas. 
Creación de nuevos SIGB para la catalogación. Es necesario concretar el diseño de la nueva generación de SIGB (Calhoun, 2006) que permita a los profesionales de las bibliotecas trabajar de forma nativa con datos bibliográficos y de autoridades en BIBFRAME. Este es un paso crítico, porque es el que va a dejar patente de forma explícita a los catalogadores el profundo cambio conceptual que representa la nueva actividad catalogadora. El profesional pasa a usar formularios que no incluyen números y códigos para los elementos -al contrario que los sistemas basados en MARC-, sino etiquetas. Los formularios de entrada se corresponden con la terminología empleada en las reglas de catalogación RDA tal y como refleja el RDA Toolkit (McCallum, 2017).

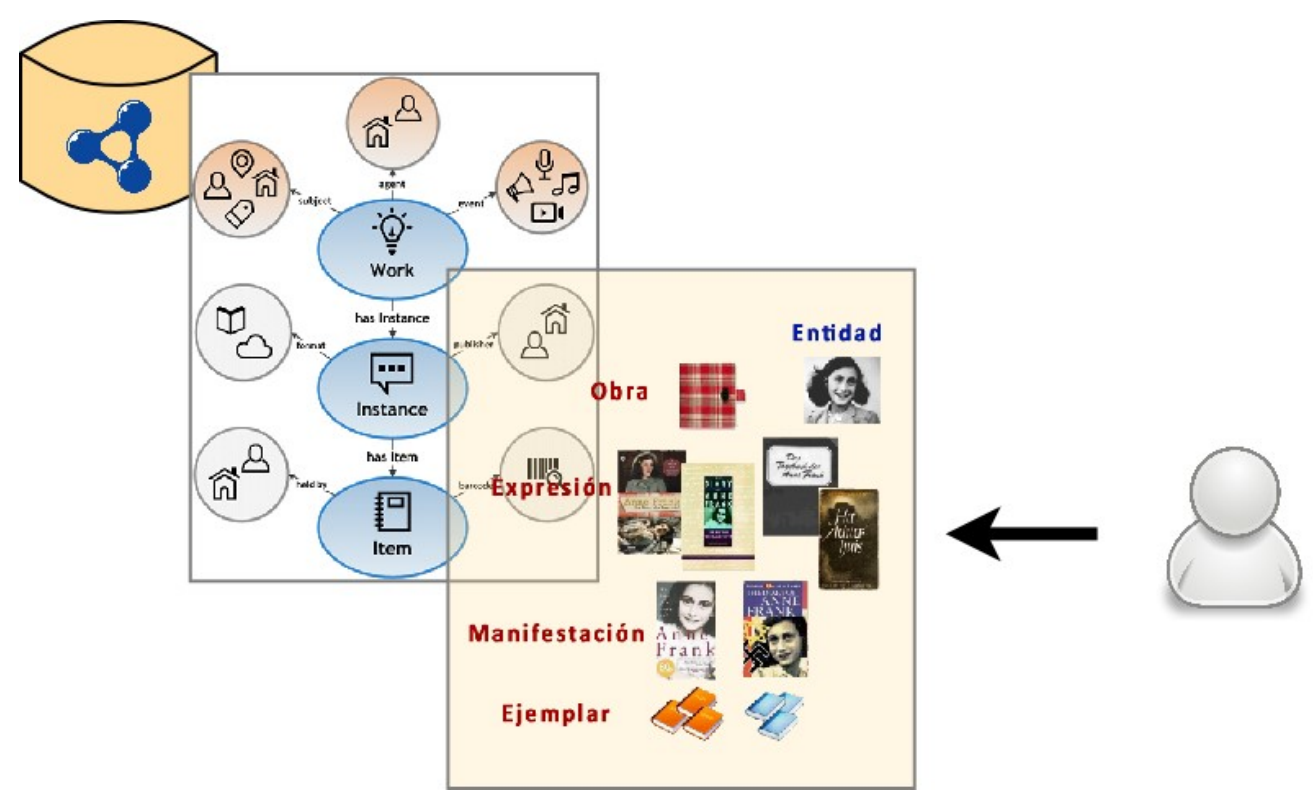

Figura 11. Editor de catalogación desde un punto de vista conceptual. Elaboración propia. 


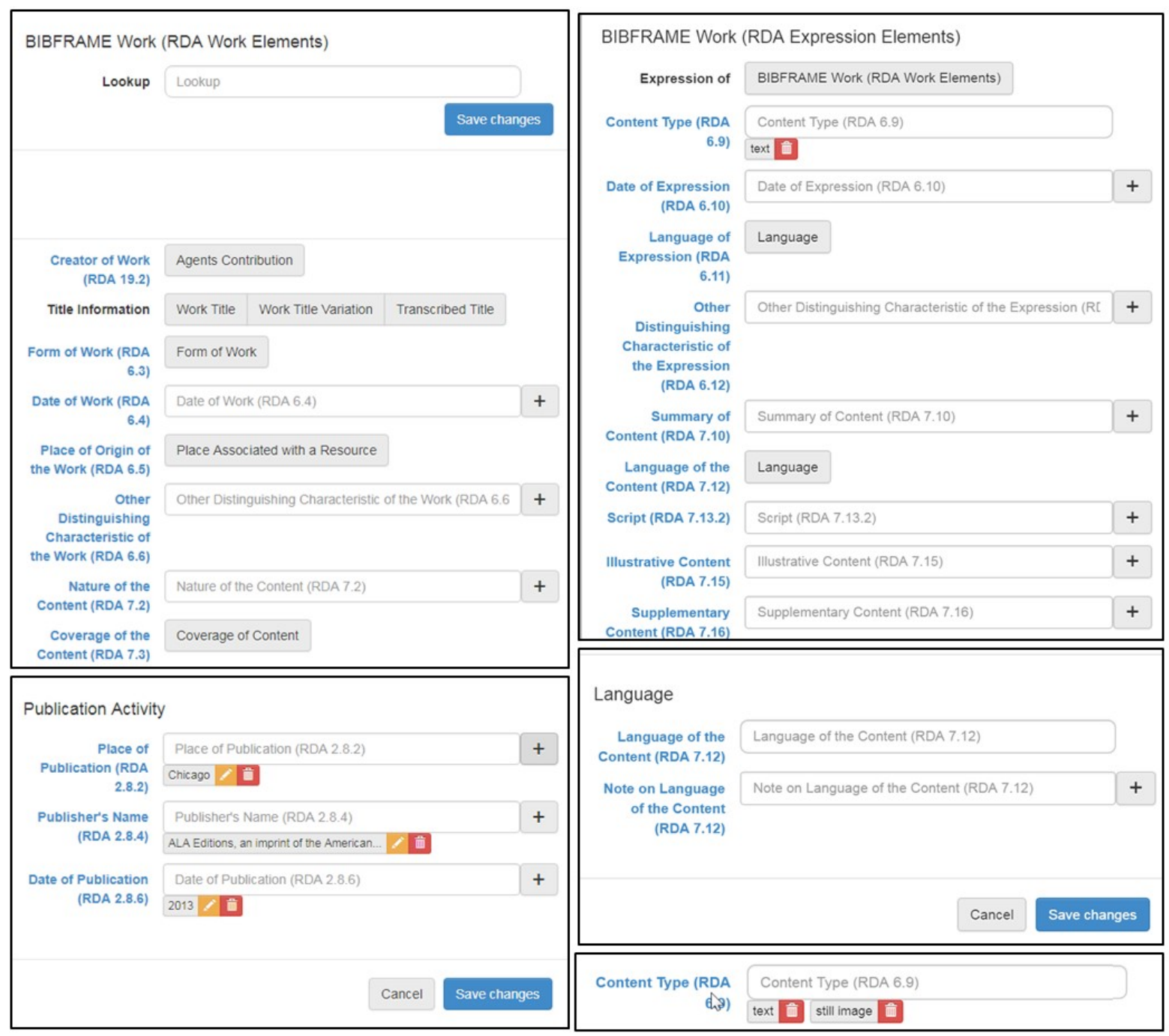

Figura 12. Editor de BIBFRAME de la fase 2 del piloto de la Library of Congress. Extraído de Carlton et al. (2017).

Los nuevos editores de catalogación planteados se basan en el modelo LRM, y en los recursos bibliográficos y entidades que propone y que inspiran las directrices RDA (Smith, Stahmer y Miller, 2016). Es necesario crear obras, expresiones, manifestaciones y ejemplares, así como agentes y sus relaciones de forma consistente, a través de los formularios propuestos. Por debajo de esta interfaz visible para el usuario estaría la implementación BIBFRAME y las relaciones entre obras, instancias, agentes y demás clases del marco bibliográfico, expresadas como tripletas RDF, que hacen posible su integración en la web semántica (Wiggins et al., 2016).

Existen iniciativas como la herramienta gratuita educacional RIMMF (RDA in Many Metadata Formats), o OliSuite/WeCat, Software de @Cult en asociación con Casalini-Libri, que pretende convertirse en un SIGB de próxima generación (Guerrini y Possemato, 2016). Pero en la actualidad todos los ojos están puestos en la fase 2 del piloto de BIBFRAME 2.0 emprendido por la LC, y de la que ya se pueden ver los primeros resultados (Carlton et al., 2017). El editor de BIBFRAME que forma parte del piloto permite la entrada de elementos de vocabulario de BIBFRAME.

En el piloto se realiza un mapeo entre los elementos de BIBFRAME (obras, instancias e ítems) y los de RDA (obras, expresiones, manifestaciones e ítems). Para completar el entorno de catalogación, incorpora una base de datos BIBFRAME de descripciones bibliográficas en la que buscar, a la que se denomina Linked Data Store y con la que se puede interactuar para realizar búsquedas. 


\subsection{Otras iniciativas}

Formación de profesionales. Son grandes cambios los que integran el paso a los nuevos estándares de catalogación, y esto implica un importante esfuerzo de formación de los profesionales de las bibliotecas. Esta formación se extiende más allá del ámbito profesional, y abarca al propio diseño del grado universitario de Información y Documentación.

Exposición de datos estructurados en Schema.org. Fuera de las fronteras bibliotecarias, motores de búsqueda y otras aplicaciones capaces de gestionar información semántica en la web lo hacen a través del vocabulario para datos estructurados Schema.org. En caso de que las organizaciones alberguen la información de sus catálogos como tripletas RDF según el formato BIBFRAME, si quieren incrustar en páginas web de sus portales parte de este contenido para su adecuado consumo por parte de los buscadores web, podrían traducir mediante mapeos de ontologías dicha información disponible en estas tripletas RDF de BIBFRAME a datos estructurados de Schema.org. De esta forma los motores de búsqueda pueden procesar y enriquecer el contenido proporcionado al usuario en los resultados de su búsqueda, recibiendo para ello la colaboración de las propias bibliotecas.

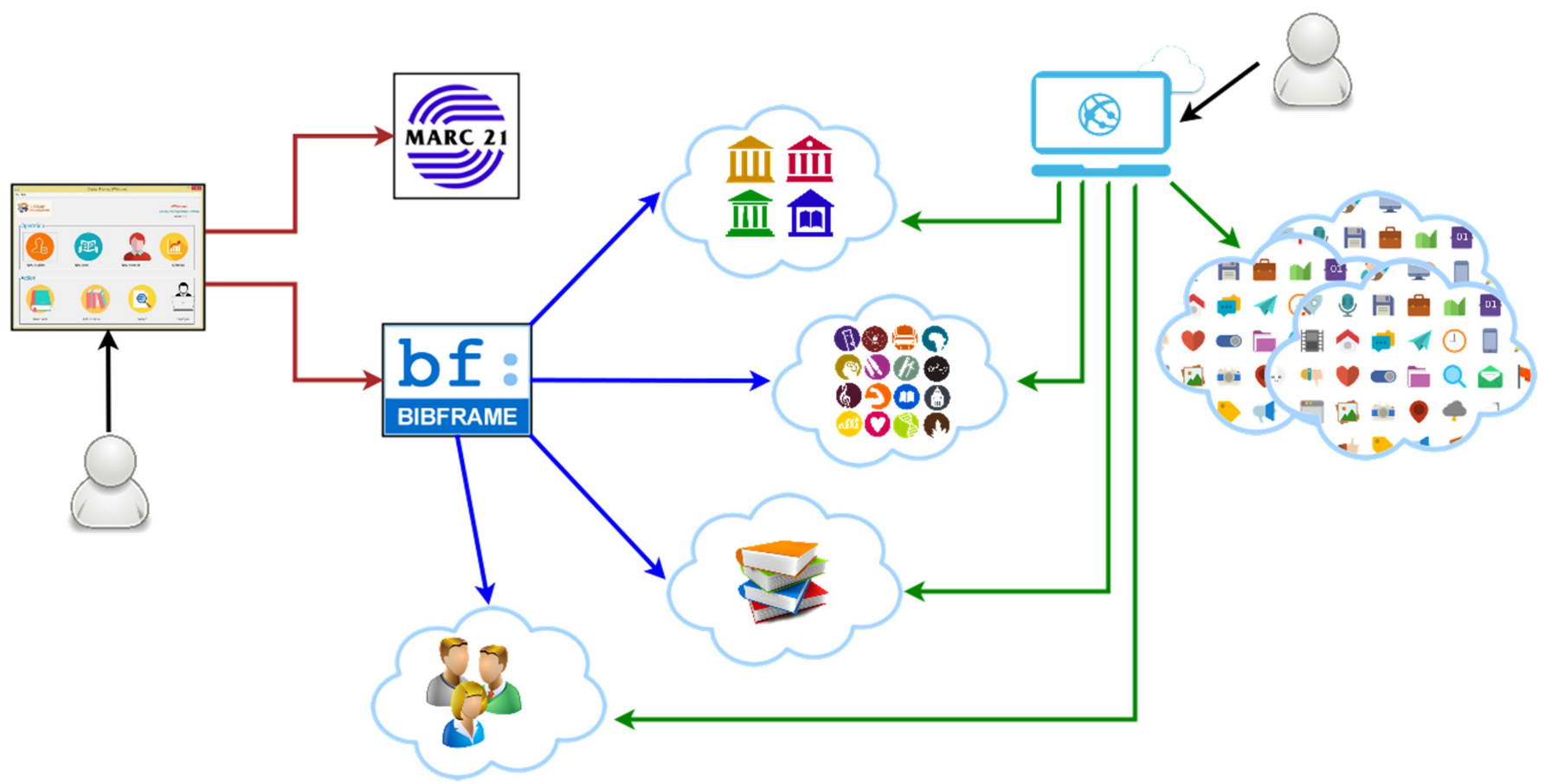

Figura 13. En el SIGB podrían convivir registros MARC con datos en BIBFRAME. Los recursos de BIBFRAME pueden residir en ubicaciones concretas de la web, mantenidas por instituciones designadas, y ser usados por otras aplicaciones, combinados con otros datos enlazados, para enriquecer la información ofrecida al usuario. Elaboración propia.

Reorganización informacional en el seno de las bibliotecas. En el contexto de catalogación clásico, los sistemas de gestión bibliotecaria almacenan localmente la práctica totalidad de la información bibliográfica y de autoridades que gestiona la institución. La evolución de las tecnologías ha permitido que estos sistemas sean capaces de obtener esta información de diversas instituciones de referencia que la comparten (LC, BNE, CSIC...), a través de protocolos como Z39.50, pero copiándola en última instancia a las bases de datos de los propios sistemas de bibliotecas. Es lo que se conoce como copy cataloging. El aterrizaje de BIBFRAME y RDF en la escena hace que obras, expresiones, agentes y otras entidades -todas ellas identificadas con una URI única- residan en la web, y que las nuevas herramientas de catalogación hagan referencia a estas. Decidir quiénes serán las entidades de referencia responsables de albergar y mantener qué recursos, y la forma en que estos se compartirán para su uso desde las herramientas de catalogación y desde otras aplicaciones destinadas al usuario final son cuestiones que todavía quedan en el tintero.

Extensiones de la ontología BIBFRAME. Hay diversas bibliotecas universitarias que estudian cómo extender la ontología de BIBFRAME para acomodarse a detalles y características de material bibliográfico específico, como cartografía, música, imagen fija o registros de audio (McCallum, 2017). Cabe destacar iniciativas como la de bibliotek-o, producto fruto del esfuerzo conjunto de los proyectos LD4L-Labs y LD4P. Las bibliotecas de las universidades españolas todavía permanecen ajenas a este tipo de iniciativas y otras relacionadas con BIBFRAME. 


\subsection{Resumen de la situación en las instituciones examinadas}

Situación actual de la catalogación en las bibliotecas. En la Tabla II se puede observar que las bibliotecas navarras (como ejemplo representativo de la mayoría de bibliotecas públicas y universitarias españolas) están a la expectativa de los avances que sobre la implantación de RDA haga la BNE. Las bibliotecas nacionales son los grandes referentes en materia de catalogación, y en la actualidad el proyecto $3 \mathrm{R}$ está posponiendo el proceso de adaptación de estas al nuevo paradigma.

\begin{tabular}{|c|c|c|c|c|c|c|c|c|}
\hline $\begin{array}{c}\text { Biblio- } \\
\text { teca }\end{array}$ & Tipo & SIGB & $\begin{array}{l}\text { Catalog. } \\
\text { tradicional }\end{array}$ & $\begin{array}{c}\text { Directrices } \\
\text { RDA }\end{array}$ & $\begin{array}{l}\text { Formato } \\
\text { codif. }\end{array}$ & $\begin{array}{c}\text { BIBFR } \\
\text { AME }\end{array}$ & $\begin{array}{c}\text { Situación } \\
\text { actual }\end{array}$ & $\begin{array}{c}\text { Portal } \\
\text { datos } \\
\text { enlazados }\end{array}$ \\
\hline b & $\begin{array}{l}\text { Biblioteca } \\
\text { pública }\end{array}$ & $\begin{array}{l}\text { AbsysNET } \\
\text { (Baratz) }\end{array}$ & $\mathrm{RC}$ e ISBD & No & MARC 21 & No & $\begin{array}{l}\text { A la espera } \\
\text { de BNE } \\
\text { (ene. 2019) }\end{array}$ & No \\
\hline 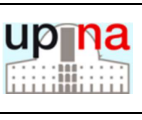 & $\begin{array}{l}\text { Biblioteca } \\
\text { universitaria }\end{array}$ & $\begin{array}{l}\text { AbsysNET } \\
\text { (Baratz) }\end{array}$ & $\mathrm{RC}$ e ISBD & $\begin{array}{c}\text { Descripciones } \\
\text { y campos } \\
\text { concretos } \\
\end{array}$ & MARC 21 & No & $\begin{array}{c}\text { A la espera } \\
\text { de BNE } \\
\text { (ene. 2019) } \\
\end{array}$ & No \\
\hline & $\begin{array}{l}\text { Biblioteca } \\
\text { universitaria }\end{array}$ & $\begin{array}{c}\text { Sierra } \\
\text { (Innovative) }\end{array}$ & $\mathrm{RC}$ e ISBD & $\begin{array}{c}\text { Descripciones } \\
\text { y campos } \\
\text { concretos } \\
\end{array}$ & MARC 21 & No & $\begin{array}{c}\text { A la espera } \\
\text { de BNE } \\
\text { (ene. 2019) }\end{array}$ & No \\
\hline & $\begin{array}{l}\text { Biblioteca } \\
\text { nacional }\end{array}$ & $\begin{array}{c}\text { Sierra } \\
\text { (Innovative) }\end{array}$ & AACR2 & Desde 2016 & MARC 21 & No & $\begin{array}{c}\text { A la espera de } \\
\text { proyecto 3R } \\
\text { (jun. 2018) }\end{array}$ & $\begin{array}{c}\text { A } \\
\text { implementar } \\
\text { en } 2018 \\
\end{array}$ \\
\hline$\frac{\sigma_{0} \theta_{3}}{B N E}$ & $\begin{array}{l}\text { Biblioteca } \\
\text { nacional }\end{array}$ & $\begin{array}{l}\text { Symphony } \\
\text { (SirsiDynix) }\end{array}$ & $\mathrm{RC}$ e ISBD & $\begin{array}{c}\text { A partir de } \\
\text { principios de } \\
2019\end{array}$ & MARC 21 & No & $\begin{array}{l}\text { A la espera de } \\
\text { proyecto 3R } \\
\text { (jun. 2018) }\end{array}$ & datos.bne.es \\
\hline & $\begin{array}{c}\text { Biblioteca } \\
\text { universitaria }\end{array}$ & $\begin{array}{c}\text { Sierra } \\
\text { (Innovative) }\end{array}$ & AACR2 & $\begin{array}{c}\text { A partir de } \\
\text { finales de } 2014\end{array}$ & MARC 21 & No & $\begin{array}{c}\text { A la espera de } \\
\text { proyecto 3R } \\
\text { (jun. 2018) }\end{array}$ & No \\
\hline
\end{tabular}

Tabla II. Resumen de la situación de la catalogación de las bibliotecas estudiadas.

Publicación de datos abiertos enlazados. La Tabla III resume las distintas iniciativas de publicación de datos abiertos enlazados acometidas por algunas de las instituciones, siendo el portal de la BNE un referente internacional. La evolución de las ontologías de BIBFRAME y de RDA Registry están marcando el progreso de estos sitios web.

\begin{tabular}{|c|c|c|c|c|}
\hline Entidad & Tipo & Producto & Ontología actual & Situación \\
\hline $\mathbf{B}$ & Biblioteca nacional & - & - & Comienzo de definición (2018) \\
\hline$\frac{\text { BNE }}{\mathrm{BNE}}$ & Biblioteca nacional & $\begin{array}{c}\text { Portal } \\
\text { datos.bne.es }\end{array}$ & $\begin{array}{c}\text { Ontología propia, basada } \\
\text { fundamentalmente en FRBR-LRM }\end{array}$ & $\begin{array}{c}\text { Posible evolución hacia la ontología de } \\
\text { RDA Registry (2018) }\end{array}$ \\
\hline
\end{tabular}

Tabla III. Resumen de la situación de publicación de datos abiertos en las entidades estudiadas.

Formación sobre RDA en las instituciones. En la Tabla IV se expone a grandes rasgos las iniciativas de formación abordadas. Además de los sitios web sobre RDA ofrecidos por las bibliotecas nacionales, cabe destacar el célebre MOOC sobre estas directrices confeccionado por la biblioteca de la UIB. En lo que a bibliotecas nacionales se refiere, mientras que la $\mathrm{BC}$ ha generado documentación propia acerca de RDA y su implantación, la BNE prefiere que los estándares y directrices alcancen un mayor grado de madurez.

\begin{tabular}{|c|c|c|c|c|c|c|}
\hline Biblioteca & Tipo & Personal formado & $\begin{array}{c}\text { Sitio sobre } \\
\text { RDA }\end{array}$ & $\begin{array}{c}\text { Material } \\
\text { propio }\end{array}$ & $\begin{array}{c}\text { Realización } \\
\text { conferencias }\end{array}$ & $\begin{array}{c}\text { Impartición } \\
\text { formación }\end{array}$ \\
\hline 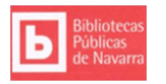 & $\begin{array}{l}\text { Biblioteca } \\
\text { pública }\end{array}$ & $\begin{array}{c}\text { En proceso } \\
\text { (3 trabajadores) }\end{array}$ & - & - & & - \\
\hline 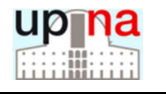 & $\begin{array}{c}\text { Biblioteca } \\
\text { universitaria }\end{array}$ & $\begin{array}{l}\text { Grupo responsable } \\
\text { (foros de formación) }\end{array}$ & - & - & & - \\
\hline Qu Univingridad & $\begin{array}{c}\text { Biblioteca } \\
\text { universitaria }\end{array}$ & $\begin{array}{c}\text { En proceso } \\
\text { (1 catalogador) }\end{array}$ & - & - & & - \\
\hline
\end{tabular}




\begin{tabular}{|c|c|c|c|c|c|c|}
\hline Biblioteca & Tipo & Personal formado & $\begin{array}{c}\text { Sitio sobre } \\
\text { RDA }\end{array}$ & $\begin{array}{c}\text { Material } \\
\text { propio }\end{array}$ & $\begin{array}{c}\text { Realización } \\
\text { conferencias }\end{array}$ & $\begin{array}{c}\text { Impartición } \\
\text { formación }\end{array}$ \\
\hline & $\begin{array}{c}\text { Biblioteca } \\
\text { nacional }\end{array}$ & $\begin{array}{l}\text { Sí (Servicio de } \\
\text { Normalización } \\
\text { Bibliográfica) }\end{array}$ & Hacia RDA & $\begin{array}{l}\text { Sí (traducciones } \\
\text { y documentos) }\end{array}$ & Sí & $\begin{array}{c}\text { Sí (encuentros } \\
\text { con bibliotecas } \\
\text { catalanas) }\end{array}$ \\
\hline$\frac{\alpha_{3}}{\mathrm{BNE}}$ & $\begin{array}{c}\text { Biblioteca } \\
\text { nacional }\end{array}$ & $\begin{array}{l}\text { En proceso avanzado } \\
\text { (Dirección Técnica de } \\
\text { Procesos y Servicios) }\end{array}$ & RDA & $\begin{array}{l}\text { Por concretar } \\
\quad(2018)\end{array}$ & Sí & $\begin{array}{l}\text { Por concretar } \\
\quad(2018)\end{array}$ \\
\hline & $\begin{array}{c}\text { Biblioteca } \\
\text { universitaria }\end{array}$ & $\begin{array}{l}\text { Sí (Servicio de } \\
\text { Catalogación) }\end{array}$ & $\begin{array}{c}\text { Catalogación } \\
\text { RDA }\end{array}$ & $\begin{array}{l}\text { Sí (documentos } \\
\text { y vídeos) }\end{array}$ & Sí & $\begin{array}{l}\text { MOOC sobre } \\
\text { RDA }\end{array}$ \\
\hline
\end{tabular}

Tabla IV. Resumen de la situación de formación en RDA en las bibliotecas estudiadas.

\section{CONCLUSIONES}

El estudio cualitativo realizado incluye como parte integral una serie de entrevistas y charlas realizadas sobre el estado del arte en materia de catalogación bibliotecaria, realizadas a una selección de instituciones implicadas de una u otra forma en el tema, y que han permitido obtener una visión representativa de qué iniciativas se están abordando y en qué situación se encuentra la adaptación a RDA y BIBFRAME en el ámbito de las bibliotecas españolas.

En base a este estudio se puede concluir que los profesionales implicados en los procesos de catalogación y en el desarrollo de herramientas y aplicaciones relacionadas con este ámbito se enfrentan a una situación ciertamente convulsa:

- En un corto lapso de tiempo, se han sucedido grandes cambios y de forma continuada en la práctica totalidad de principios, modelos, directrices, entornos, ontologías, herramientas y estándares que sustentan la nueva filosofía de catalogación.

- Las instituciones responsables de distintos estándares - principalmente la LC (garante de la adecuada evolución de BIBFRAME) y el RSC (encargado del desarrollo de RDA), no siempre colaboran estrechamente para un desarrollo concomitante entre éstos.

- La dicotomía entre el vocabulario de BIBFRAME (sus clases, propiedades y relaciones) y el de RDA (sus entidades, atributos y relaciones) ha dado pie a posturas encontradas entre profesionales de bibliotecas sobre qué ontología usar a la hora de publicar los catálogos como datos abiertos enlazados. En la actualidad, parece haber indicios de una cierta polarización entre RDA Registry (ontología gestionada por el RDA Steering Committee y BIBFRAME 2.0 (ontología concebida por la Library of Congress).

Frente a este ecosistema inhóspito y beligerante, ciertamente es difícil para la comunidad bibliotecaria apostar por emprender acciones para avanzar hacia la nueva era de la catalogación. Pero es algo fundamental. No hay mejor manera de aprender qué quiere y necesita el usuario final que a través de la experiencia. Es necesario ofrecerle propuestas, darle una referencia motivada de qué se pretende, e interpretar su reacción y opiniones. Y obrar en consecuencia y seguir avanzando. Afortunadamente, se trata de acometer un proceso iterativo, facilitado en los primeros pasos a dar por:

- La buscada independencia existente entre estándares, especialmente entre contenido (normas y directrices) y continente (entorno y formato de codificación) catalográficos.

- La actual flexibilidad que ofrecen las herramientas y sistemas de gestión de bibliotecas actuales, que permiten incorporar nuevos campos, diseñar plantillas y establecer validaciones para editar la información de catalogación (aunque siempre manteniendo el formato de codificación).

Las bibliotecas nacionales y algunas bibliotecas universitarias del Estado están abriendo la senda del cambio y abordando transformaciones profundas hacia RDA y hacia los datos enlazados, sin que esta circunstancia suponga un hecho traumático, gracias a que:

- La implementación de RDA de momento se está abordando sobre el formato de codificación MARC 21, el mismo que el empleado en las normas de catalogación tradicionales de AACR2, ISBD y RC españolas. Esto supone cambios formales en el contenido y modificación de campos y códigos de subcampo de los registros catalográficos, algo fácilmente gestionable con los SIGB actualmente usados en bibliotecas.

- La publicación de datos abiertos enlazados en la webse está afrontando con un amplio margen para elegir vocabulario y ontología de las tripletas RDF.

Hay que reconocer que los beneficios del nuevo paradigma de catalogación serán más patentes cuando BIBFRAME entre en escena con un papel protagonista en la función. Así, los datos enlazados serán parte inherente del entorno de gestión bibliotecaria y dejarán de pergeñarse mediante transformaciones de la información almacenada en los repositorios de las bibliotecas. 
Todavía no parece un paso a dar de forma inminente, y sin dudava a ser mucho más dificultoso que el anterior, porque el cambio en el entorno y formato obliga a:

- Una profunda revisión de los editores de catalogación (aún en fase piloto),

- Una cuidada planificación de la migración de los registros retrospectivos (cuyas herramientas están siendo perfeccionadas, y que en muchos casos no podrán automatizar completamente el proceso), y

- La más que previsible convivencia de registros previos codificados en formato MARC 21 y registros actuales como tripletas RDF (circunstancia que todavía es necesario estudiar cómo afrontar, buscando minimizar las implicaciones para el usuario final).

Este proceso del cambio tiene que ser aprovechado para homogeneizar las prácticas de catalogación. En el caso de España, aquellas bibliotecas que utilizan las RC españolas y aquéllas que optan por las AACR2 pasan a unificar sus directrices fundamentales. Ante tal circunstancia, los criterios convergerían. No obstante, el gran margen que da RDA a las bibliotecas para establecer la asignación de determinados valores bibliográficos para los distintos materiales -que se traduce en la definición de sus correspondientes perfiles RDA- puede dar lugar a diferencias de criterio, que habría que tratar de recortar en aras de esa pretendida homogeneidad y facilitar el enlace de datos entre instituciones.

Dado el panorama actual, es de vital interés continuar este estudio en los próximos años y analizar cualitativamente los avances del nuevo marco bibliográfico en las instituciones. La forma en que evolucione el estándar y su relación con las directrices de catalogación marcarán los designios de la comunidad bibliotecaria y su verdadero protagonismo a través de la publicación en la web del contenido informativo que albergan. Ante esta circunstancia, el profesional bibliotecario debe progresar en sus habilidades y capacidades, y recibir formación no solo en meras cuestiones de normativa, sino buscando también ahondar en la idiosincrasia de la web semántica y los datos enlazados, que van a marcar indefectiblemente el definitivo paradigma catalogador de las próximas décadas.

Actualmente no siempre se percibe de forma evidente los beneficios logrados en los entornos de producción de las bibliotecas, más si cabe cuando la hoja de ruta establece una evolución prolongada en el tiempo de la que todavía se están dando los primeros pasos. Esto dificulta calibrar el grado de éxito de los avances realizados hasta la fecha. Los usuarios todavía no ven mejorada ostensiblemente su experiencia en su relación con el catálogo con la llegada de RDA. Esto llegará después, cuando BIBFRAME se integre como entorno de intercambio bibliográfico.

Las características y funcionalidades que presente la nueva generación de SIGB van a jugar un papel clave, y también van a influir de forma determinante en otro ámbito digno de estudio, como es la automatización de la catalogación. En efecto, la enorme cantidad de material documental que se genera hoy en día proveniente de editores, autores y otras fuentes obligan a contemplar soluciones en este sentido, que presumiblemente se verán simplificadas por la llegada del nuevo marco bibliográfico, fundamentado en los datos enlazados.

Hay todavía incógnitas en el trayecto, y el cronograma no está definido al detalle, pero la meta es clara, y llegar a ella es cuestión de tiempo, voluntad y esfuerzo de todas las partes.

\section{BIBLIOGRAFÍA}

AGENJO-BULLÓN, X. y HERNÁNDEZ-CARRASCAL, F. Avances de Bibframe en 2014: perspectivas del nuevo modelo bibliográfico. Anuario ThinkEPI, 2015, vol. 9, p. 269-277. Disponible en: https://doi.org/10.3145/thinkepi.2015.64.

AGENJO-BULLÓN, X. y HERNÁNDEZ-CARRASCAL, F. Avances de Bibframe en 2016: perspectivas del nuevo modelo bibliográfico. Anuario ThinkEPI, 2017, vol. 11, p. 310-318. Disponible en: https://doi.org/10.3145/thinkepi.2017.58.

ALEMU, G.; STEVENS, B.; ROSS, P. y CHANDLER, J. Linked Data for libraries. New Library World [en línea].2012, vol. 113, no 11/12, p. 549-570. Disponible en: https://doi.org/10.1108/03074801211282920.

ALIADA PROJECT CONSORTIUM. Automatic Publication under Linked Data Paradigm of Library Data: ALIADA, an Open Source Solution to Easily Publish Linked Data of Libraries and Museums. SWIB15, Hamburg, nov. 2015. Disponible en: <https://es.slideshare.net/aliadaproject/swib15-aliada> [Consulta: 24 de febrero de 2018].

AMMERMAN, J. 2016. Publishing Bibliographic Records from Alma to the Library Link Network. IGeLU and ELUNA Show\&Tell. Disponible en: <https://www.youtube.com/watch?v=ymDgBtS_6EE $>$ [Consulta: 24 de febrero de 2018].

BNE, 2016. Estructura (GARR/GSARE). En Publicaciones de la Biblioteca Nacional de España. Manual de Autoridades [en línea]. Disponible en: 
$<$ http://www.bne.es/es/Micrositios/Publicaciones/AUTORIDADES/004_Estructura> [Consulta: 24 de febrero de 2018].

CALHOUN, K. The Changing Nature of the Catalog and its Integration with Other Discovery Tools. Journal of Library Metadata [en línea]. 2006, vol. 8, $\mathrm{n}^{\mathrm{o}}$ 2, p. 169-197. Disponible en: https://doi.org/10.1080/10911360802087374.

CARLTON, T.; HAWKINS, L.; FRANK, P. E INSTRUCTIONAL PROGRAMS DIVISION, 2017. Library of Congress BIBFRAME Pilot 2 Training for Catalogers. Module 2: The BIBFRAME Editor and de BIBFRAME Linked Data Store. Library of Congress [en línea]. Disponible en: $<$ https:/www.loc.gov/catworkshop/bibframe/Training/Bf2.0/Bf2TrainingManual.docx> [Consulta: 24 de febrero de 2018].

COYLE, K.The Library Catalog: Some Possible Futures. The Journal of Academic Librarianship [en línea].2007, vol 33, $\mathrm{n}^{\circ}$ 3, p. 414-416. Disponible en: https://doi.org/10.1016/j.acalib.2007.03.001.

DULL, M.E.Moving metadata forward with BIBFRAME: An interview with Rebecca Guenther. Serials Review, 2016, vol. 42, no 1, p. 65-69. Disponible en: https://doi.org/10.1080/00987913.2016.1141032.

FERRAN, I. Implementación de RDA en las bibliotecas de Cataluña. Desarrollo de RDA y casos de implementación en Europa. Cita en la BNE, Madrid, oct. 2017. Disponible en: $<$ http://www.bne.es/es/ComunidadBNE/Retransmisiones/1027-Jornada-DesarrollodeRDAycasos $>$ [Consulta: 24 de febrero de 2018].

FURRIE, B. 2009. Understanding MARC Bibliographic: Machine-Readable Cataloging. Eighth edition. Washington: Library of Congress [en línea]. Disponible en: <http://www.loc.gov/marc/umb/> [Consulta: 24 de febrero de 2018].

GALEFFI, A.; BERTOLINI, M.V.; BOTHMANN, R.L.; ESCOLANO RODRÍGUEZ, E. y MCGARRY, D. 2016. Statement of International Cataloguing Principles (ICP). IFLA [en línea]. Disponible en: $<$ https://www.ifla.org/files/assets/cataloguing/icp/icp_2016-en.pdf $>$ [Consulta: 24 de febrero de 2018].

GUERRINI, M. y POSSEMATO, T. From record management to data management: RDA and new application models BIBFRAME, RIMMF, and OliSuite/WeCat. Cataloging and Classification Quarterly, 2016, vol. 54, $\mathrm{n}^{\circ} 3$, p. 179-199. Disponible en: https://doi.org/10.1080/01639374.2016.1144667.

GUHA, R.V.; BRICKLEY, D. y MACBETH, S.Schema.org: Evolution of the Structured Data on the Web. Communications of the ACM, 2015, vol. 59, $\mathrm{n}^{\mathrm{o}}$ 2, p. 44-51. Disponible en: https://doi.org/10.1145/2844544.

HUTHWAITE, A. Preface. En Joint Steering Commitee. Anglo-American Cataloguing Rules: Second Edition: 2002 Revision. Chicago: American Library Association, 2002.

IFLA. 2009. Declaración de Principios Internacionales de Catalogación. IFLA [en línea]. Disponible en: $<$ https://www.ifla.org/files/assets/cataloguing/icp/icp_2009-es.pdf $>$ [Consulta: 24 de febrero de 2018].

LIBRARY OF CONGRESS. 2008. On the record. Working Group on the Future of Bibliographic Control [en línea] Disponible en: <https://www.loc.gov/bibliographic-future/news/lcwg-ontherecord-jan08-final.pdf $>$ [Consulta: 24 de febrero de 2018].

LIBRARY OF CONGRESS. 2017. Overview of the BIBFRAME Model. Library of Congress [en línea]. Disponible en: $<$ https://www.loc.gov/bibframe/docs/bibframe2-model.html $>$ [Consulta: 24 de febrero de 2018].

MCCALLUM, S.H. BIBFRAME Development. JLIS.it [en línea]. 2017, vol. 8, no 3, p. 71-85. Disponible en: https://dx.doi.org/10.4403/jlis.it-12415.

MERING, M.The RDA Workbook: Learning the Basics of Resource Description and Access. Santa Barbara, California: Libraries Unlimited, 2014.

MILLER, E. 2015. The Libhub Initiative: Increasing the Web Visibility of Libraries. DCMI / ASIS\&T Webinar. Diponible en: $<$ https://www.youtube.com/watch?v=dfDd6N9xGLY> [Consulta: 24 de febrero de 2018].

PARK, T.K. y MORRISON, A.M.The nature and characteristics of bibliographic relationships in RDA cataloging records in OCLC at the beginning of RDA implementation. Cataloging and Classification Quarterly, 2017 vol. 55 , $n^{\circ}$ 6, p. 361-386. Disponible en: https://doi.org/10.1080/01639374.2017.1319451.

PESCH, O. y MILLER, E. Using BIBFRAME and Library Linked Data to Solve Real Problems: An Interview with Eric Miller of Zepheira. Serials Librarian, 2016, vol. 71, $\mathrm{n}^{\mathrm{o}}$ 1, p. 1-8. Disponible en: https://doi.org/10.1080/0361526X.2016.1183159.

RDA STEERING COMMITTEE. 2017. About RDA. RSC [en línea]. Disponible en: <http://www.rdarsc.org/content/about-rda> [Consulta: 24 de febrero de 2018].

RDF WORKING GROUP. 2014. Resource Description Framework (RDF). W3C [en línea]. Disponible en: $<$ https://www.w3.org/RDF/> [Consulta: 24 de febrero de 2018].

RIVA, P.; LE BOEUF, P. y ZUMER, M. 2017. IFLA Library Reference Model: A Conceptual Model for Bibliographic Information. IFLA [en línea]. Disponible en: $<$ https://www.ifla.org/files/assets/cataloguing/frbrlrm/ifla-lrm-august-2017.pdf> [Consulta: 24 de febrero de 2018]. 
SANTOS, R. 2017. Implementación de RDA en la BNE. Desarrollo de RDA y casos de implementación en Europa. Cita en la BNE, Madrid, oct. 2017. Disponible en: < http://www.bne.es/es/ComunidadBNE/Retransmisiones/1027Jornada-DesarrollodeRDAycasos> [Consulta: 24 de febrero de 2018].

SMITH, M.; STAHMER, C.G. y MILLER, E. 2015. BIBFLOW: A Roadmap for Library Linked Data Implementation. Coalition for Networked Information (CNI) Spring 2015 Membership Meeting, Seattle, Washington. Disponible en: $<$ https://www.youtube.com/watch?v=Z-g_yJ1FL0U\&list=PLyewpvLG0y9368fBLLO6HQ8o0z9WIbyn\&index=55> [Consulta: 24 de febrero de 2018].

SPROCHI, A. Where Are We Headed? Resource Description and Access, Bibliographic Framework, and the Functional Requirements for Bibliographic Records Library Reference Model. International Information and Library Review,2016, vol. 48, no 2, p. 129-136. Disponible en: https://dx.doi.org/10.1080/10572317.2016.1176455.

SUOMINEN, O. 2016. From MARC silos to Linked Data silos? SWIB16, Bonn, nov. 2016. Disponible en: $<$ https://www.youtube.com/watch?v=xp-PRtdF5_U> [Consulta: 24 de febrero de 2018].

TENNANT, R. 2002. MARC must die. Library Journal [en línea]. ISSN 0363-0277. Disponible en: <http://lj.libraryjournal.com/2002/10/ljarchives/marc-must-die/\#_> [Consulta: 24 de febrero de 2018].

THARANI, K. Linked Data in Libraries: A Case Study of Harvesting and Sharing Bibliographic Metadata with BIBFRAME. Information Technology \& Libraries [en línea]. 2015, vol. 34, $\mathrm{n}^{\mathrm{o}}$ 1, p. 5-19. Disponible en: https://doi.org/10.6017/ital.v34i1.5664.

UIB. 2016. El proceso de implementación de RDA en el Servei de Biblioteca i Documentació de la Universitat de les Illes Balears. UIB. Servicio de Biblioteca y Documentación [en línea]. Disponible en: $<$ http://biblioteca.uib.cat/digitalAssets/385/385092_implementacion_RDA_en_SBD_UIB_Imprimible.pdf $>$ [Consulta: 24 de febrero de 2018].

WIGGINS, B.; MCCALLUM, S.H.; HESS, K.; FRANK, P. y CANNAN, J.P. 2016. BIBFRAME on the Move. The Library of Congress Webcasts. Disponible en: $<$ https://www.youtube.com/watch?v=6Y673gCZ0ss $>$ [Consulta: 24 de febrero de 2018]. 


\begin{abstract}
Anexo
Relación de preguntas realizadas a las instituciones

Cada una de las entidades tiene su propia realidad, rol y objetivos dentro del contexto de la nueva filosofía de catalogación en las bibliotecas. Esto ha hecho conveniente seleccionar y adaptar las preguntas a hacer a sus interlocutores a las propias circunstancias de cada organización. A continuación se muestra el cuestionario completo original que ha servido como guía básica para encauzar las distintas entrevistas realizadas:
\end{abstract}

\title{
RDA y MARC21
}

- ¿Actualmente se está abordando la evolución hacia RDA manteniendo MARC 21?

- ¿Ha representado mucho cambio en los registros?

- ¿Se abordan relaciones FRBR-LRM del tipo obra-obra, obra-expresión, etc., o de momento se reducen a los de recurso bibliográfico con autoridad?

\section{Herramientas}

- ¿Han cambiado los SIGB que se utilizan, o continúan los mismos?

- ¿Ha habido que evolucionarlos, para reflejar los cambios en MARC para el paso de AACR2/RC a RDA?

- ¿Tiene el editor de catálogo una orientación FRBR-LRM (con obras, expresiones, manifestaciones e ítems), o por el contrario sigue siendo una relación de campos agrupados en áreas?

- ¿Los usuarios ven alguna diferencia a través de los OPAC y las distintas herramientas de descubrimiento?

Migración

- ¿Se ha procedido a la migración de los recursos antiguos, o conviven registros pre-RDA con registros RDA?

- ¿Se ha utilizado alguna herramienta de migración de uno a otro formato MARC?

Relaciones con otras instituciones

- ¿Hay algún tipo de relación de interdependencia con otras instituciones para avanzar en este tema?

- ¿Se está realizando alguna colaboración con empresas externas para abordar el proyecto?

BIBFRAME

- ¿Está entrando de alguna manera BIBFRAME en la ecuación o se está posponiendo su análisis?

- ¿Está BIBFRAME en algún lugar dentro de la hoja de ruta actual?

- ¿Pensáis que la incorporación de BIBFRAME va a suponer un cambio traumático, más que el paso de AACR2/RC a RDA manteniendo MARC 21?

- ¿Va a suponer la mejora definitiva para integrar los datos bibliográficos con la web, o ya con RDA se facilita esta exposición de datos enlazados?

\section{Los datos abiertos}

- ¿Se está abordando algún proyecto para la publicación de los datos del catálogo en abierto en la web?

- ¿Se ha inspirado en algún otro proyecto?

- ¿A qué ontología se ha recurrido (una existente, una propia basada en otra...)?

- ¿Es necesario hacer de momento un doble trabajo de incorporar en el catálogo y publicar en datos abiertos, o el hecho de incorporar registros en el catálogo tiene directamente su reflejo en la publicación en datos abiertos?

\section{Formación}

- ¿Se está formando mucha gente en RDA y BIBFRAME en la institución?

- ¿Creéis conviene esperar antes de aprender ciertas piezas del puzle (BIBFRAME, RDF...) a la espera de que se aclare el panorama y deje de ser tan convulso?

- ¿Va a cambiar mucho el perfil del catalogador?

- ¿Y la formación universitaria (Grado de Información y Documentación)?

Aspectos organizativos

- ¿Va a ser necesario una mayor colaboración con el departamento de informática, al haber un mayor uso de la web, herramientas para migración de datos y de nuevos estándares relacionados con la web semántica?

- ¿Habría que barajar constituirse en CRAI?

Ubicación de los datos

- En relación con la vocación de ofrecer datos enlazados, ¿creéis que en el futuro va a haber datos que van a compartirse directamente en internet (desde las distintas bibliotecas nacionales del Estado, o la Library of Congress), en vez de almacenarse localmente en el SIGB (copy-editors), como ocurre en la actualidad?

- Es decir, en vez de capturar los registros de autoridad con el protocolo Z39.50 u otros servicios web, ¿se barajará trazar una referencia o vínculo a los mismos?

Ventajas 
- ¿Se ven claras desde las bibliotecas las ventajas de la incorporación de los nuevos estándares de catalogación respecto a los existentes (en cuestiones de adquisición, catalogación, incorporación de datos de autoridad...)?

- ¿Creéis que permitirá recuperar el terreno perdido respecto a los usuarios (estudiantes, profesores o investigadores que buscan en Google Académico antes que en las herramientas de descubrimiento ofrecidas por las bibliotecas, especialmente universitarias)?

- ¿Realmente va a haber una gran exposición de los datos bibliográficos de las bibliotecas en internet, o seguirá habiendo un cierto aislamiento (por la diferente forma que cada entidad publica sus datos abiertos)?

Problemas

- ¿Cuáles consideráis que van a ser los principales problemas en la implementación de RDA y -sobre todoBIBFRAME en las bibliotecas (migración desde MARC, formación, forma de ver la catalogación de los catalogadores existentes)?

- ¿Va a ser necesaria una mayor colaboración entre bibliotecas, para fomentar la compartición de recursos o simplemente se tratará de una mayor dependencia de las grandes entidades (BNE, BC, LC...), que supuestamente son las que tendrán los principales recursos bibliográficos y datos de autoridad? 